\title{
TECHNOLOGY DEVELOPMENT FOR IRON FISCHER-TROPSCH CATALYSIS
}

Contract No. DE-AC22-94PC94055

Quarterly Technical Progress Report No. 12

Covering the Period July 1, 1997 to September 30, 1997

\author{
Prepared for \\ U.S. Department of Energy \\ Federal Energy Technology Center \\ FETC Project Manager: Richard E. Tischer \\ P. O. Box 10940 \\ Pittsburgh, PA 15236-0940
}

\author{
Submitted By \\ Project Manager: Burtron H. Davis \\ University of Kentucky Research Foundation \\ Kinkead Hall \\ Lexington, KY 40506-0057
}

Revised and Accepted

December 16, 1997 


\section{Disclaimer}

"This report was prepared as an account of work sponsored by an agency of the United States Government. Neither the United States Government nor any agency thereof, nor any of their employees, makes any warranty, express or implied, or assumes any legal liability or responsibility for the accuracy, completeness, or usefulness of any information, apparatus, product, or process disclosed, or represents that its use would not infringe privately owned rights. Reference herein to any specific commercial product, process, or service by trade name, trademark, manufacturer, or otherwise does not necessarily constitute or imply its endorsement, recommendation or favoring by the United States Government or any agency thereof. The views and opinions of authors expressed herein do not necessarily state or reflect those of the United States Government or any agency thereof." 


\section{Abstract}

The goal of the proposed work is the development of iron-based FischerTropsch catalysts that combined high activity, selectivity and life with physical robustness for slurry phase reactors that will produce either low-alpha or high-alpha products. The catalyst that is developed will be suitable for testing at the Advanced Fuels Development Facility at LaPorte, Texas or similar sized plant. Previous work by the offeror has produced a catalyst formulation that is 1.5 times as active as the "standard-catalyst" developed by German workers for slurry phase synthesis. The proposed work will optimize the catalyst composition and pretreatment operation for this low-alpha catalyst. In parallel, work will be conducted to design a high-alpha iron catalyst that is suitable for slurry phase synthesis. Studies will be conducted to define the chemical phases present at various stages of the pretreatment and synthesis stages and to define the course of these changes. The oxidation/reduction cycles that are anticipated to occur in large, commercial reactors will be studied at the laboratory scale. Catalyst performance will be determined for catalysts synthesized in this program for activity, selectivity and aging characteristics. 


\section{Table of Contents}

Page

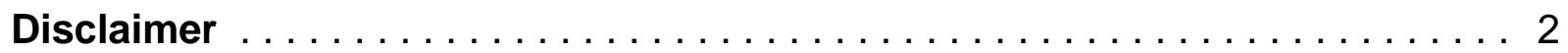

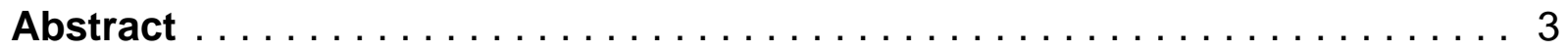

Table of Contents $\ldots \ldots \ldots \ldots \ldots \ldots \ldots \ldots \ldots \ldots \ldots \ldots \ldots \ldots$

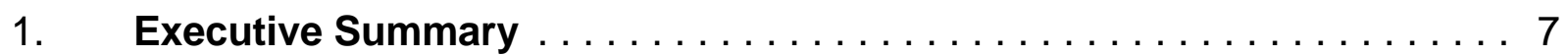

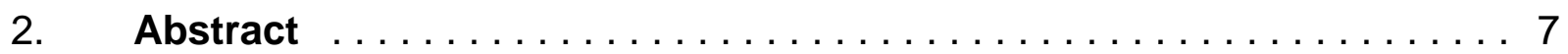

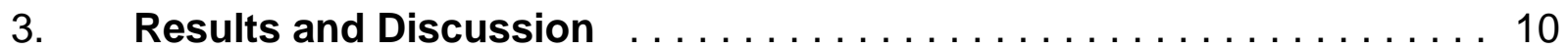

3.1. Iron Catalyst Preparation $\ldots \ldots \ldots \ldots \ldots \ldots \ldots \ldots \ldots \ldots$

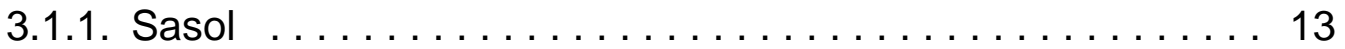

3.1.2. Ruhrchemie $\ldots \ldots \ldots \ldots \ldots \ldots \ldots \ldots \ldots \ldots \ldots \ldots \ldots$

3.1.3. The "Reichsamt" Comparative Experiments ........... 14

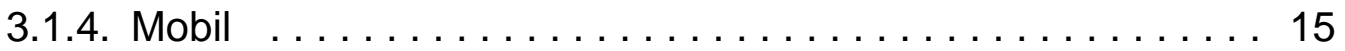

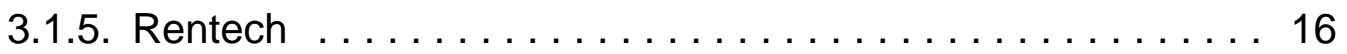

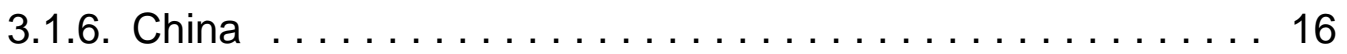

3.2. Iron Catalyst Activation $\ldots \ldots \ldots \ldots \ldots \ldots \ldots \ldots \ldots$

3.2.1. The "Reichsamt" Comparative Experiments ......... 16

3.2.2. Rentech $\ldots \ldots \ldots \ldots \ldots \ldots \ldots \ldots \ldots \ldots \ldots \ldots \ldots$

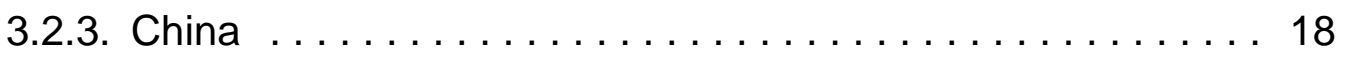

3.2.4. CAER $\ldots \ldots \ldots \ldots \ldots \ldots \ldots \ldots \ldots \ldots \ldots \ldots \ldots \ldots \ldots \ldots \ldots \ldots$

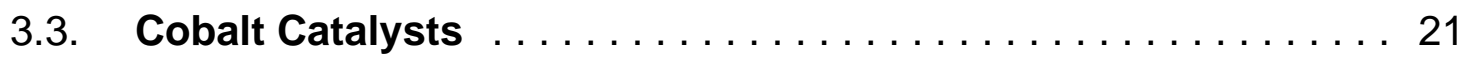

3.4. Comparison of Cobalt and Iron Catalysts ........... 24

3.5. Slurry Reactor Studies $\ldots \ldots \ldots \ldots \ldots \ldots \ldots \ldots \ldots \ldots$

3.5.1. German .................... 28 
3.5.1.1. Roelen ................... 28

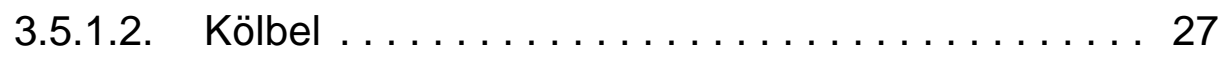

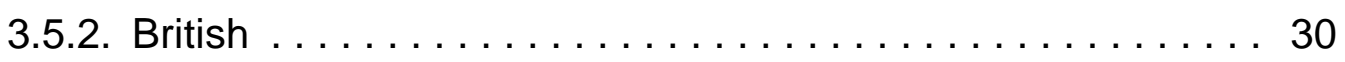

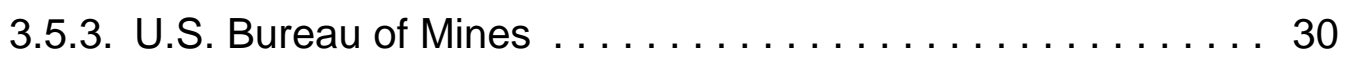

3.5.4. Mobil $\ldots \ldots \ldots \ldots \ldots \ldots \ldots \ldots \ldots \ldots \ldots \ldots \ldots \ldots \ldots \ldots \ldots$

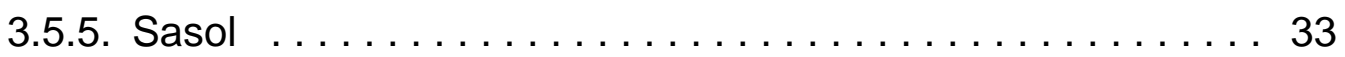

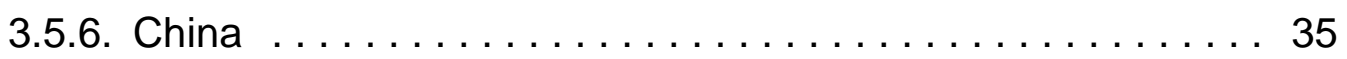

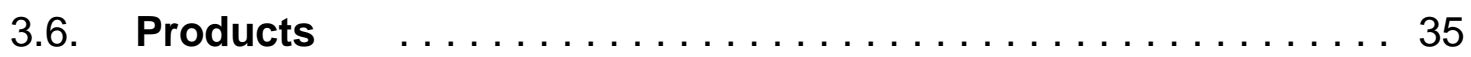

3.6.1. Low Temperature Vs. High Temperature Operation .... . 36

3.6.2. Deviations from Anderson-Schulz-Flory Distributions .... 39

3.6.2.1. Positive Deviations .............. 38

3.6.2.2. Positive Deviation at Higher Carbon Number . . . . 41

3.6.3. Cut Off of Product Distribution $\ldots \ldots \ldots \ldots \ldots \ldots \ldots 41$

3.6.3.1. Chinese $\ldots \ldots \ldots \ldots \ldots \ldots \ldots \ldots \ldots \ldots \ldots$

3.6.3.2. Syntroleum $\ldots \ldots \ldots \ldots \ldots \ldots \ldots \ldots \ldots \ldots$

3.7. Slurry-Wax/Catalyst Separation $\ldots \ldots \ldots \ldots \ldots \ldots \ldots \ldots 43$

3.7.1. British $\ldots \ldots \ldots \ldots \ldots \ldots \ldots \ldots \ldots \ldots \ldots \ldots \ldots \ldots$

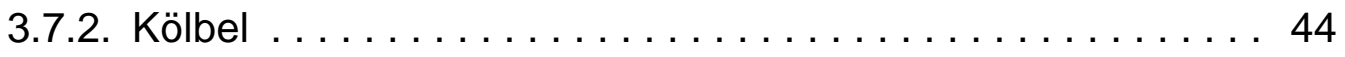

3.7.3. U.S. Bureau of Mines .................. 44

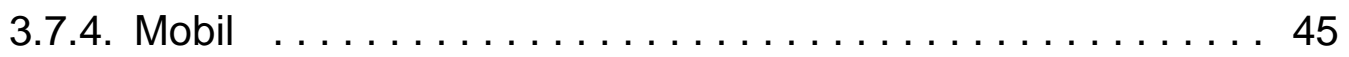

3.7.5. Sasol $\ldots \ldots \ldots \ldots \ldots \ldots \ldots \ldots \ldots \ldots \ldots \ldots \ldots$

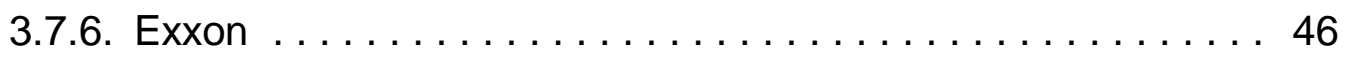

3.7.7. Statoil $\ldots \ldots \ldots \ldots \ldots \ldots \ldots \ldots \ldots \ldots \ldots \ldots \ldots$

3.7.8. China $\ldots \ldots \ldots \ldots \ldots \ldots \ldots \ldots \ldots \ldots \ldots \ldots \ldots$ 


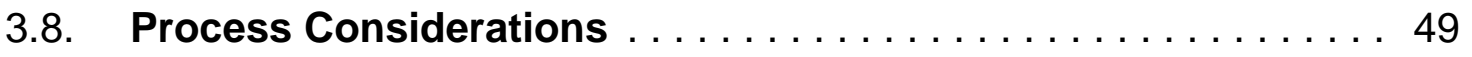

3.9. Supercritical Phase Fischer-Tropsch Synthesis $\ldots \ldots \ldots \ldots 5$

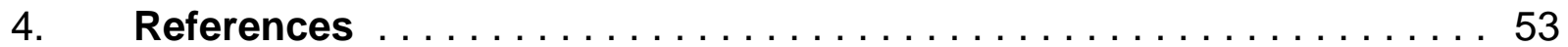

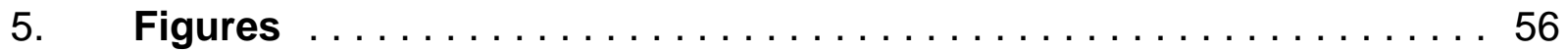




\section{Executive Summary}

A technical assessment of the Fischer-Tropsch synthesis was completed.

\section{Abstract}

The objective of this research project is to develop the technology for the production of physically robust iron-based Fischer-Tropsch catalysts that have suitable activity, selectivity and stability to be used in the slurry phase synthesis reactor development. The catalysts that are developed shall be suitable for testing in the Advanced Fuels Development Facility at LaPorte, Texas, to produce either low- or high-alpha product distributions. Previous work by the offeror has produced a catalyst formulation that is 1.5 times as active as the "standard-catalyst" developed by German workers for slurry phase synthesis. The proposed work will optimize the catalyst composition and pretreatment operation for this low-alpha catalyst. In parallel, work will be conducted to design a high-alpha iron catalyst this is suitable for slurry phase synthesis. Studies will be conducted to define the chemical phases present at various stages of the pretreatment and synthesis stages and to define the course of these changes. The oxidation/reduction cycles that are anticipated to occur in large, commercial reactors will be studied at the laboratory scale. Catalyst performance will be determined for catalysts synthesized in this program for activity, selectivity and aging characteristics.

The research is divided into four major topical areas: (a) catalyst preparation and characterization, (b) product characterization, (c) reactor operations, and (d) data assessment.

To accomplish the objectives of the project, these topics have been organized into the following technical tasks: 
a. Task 1.0 Development of Optimum Promoter Levels for Low- and High-Alpha Catalysts

The goal of this task is to identify and optimize procedure for the preparation of iron-based catalysts that combine high activity selectivity and life with physical robustness. Each of the subtasks address an area of considerable uncertainty in the synthesis of catalysts.

1.1 Determine Optimized Synthesis Procedure for High-Alpha Iron-Based Fischer-Tropsch Catalysts

- Role of precursor particle size on activity.

- Role of Cu in precipitated catalysts.

- Define attrition resistance.

1.2 Prepare Catalysts that can be Used to Determine the Role of Promoters for Low- and High-Alpha Catalysts

- $\quad$ Define optimum $\mathrm{SiO}_{2}$.

- Define optimum $\mathrm{Al}_{2} \mathrm{O}_{3}$.

1.3 Prepare Catalysts that can be Used to Quantify the Role of K on Product Selectivity in both Low- and High-Alpha Catalysts.

1.4 Complete the Optimization of the Two Best Low-Alpha, Iron-Based Fischer-Tropsch Catalysts Developed during the Previous Contract.

b. Task 2.0 Definition of Preferred Pretreatment for both Low- and High-Alpha Fischer-Tropsch Catalysts.

The goals of this task are to define the preferred treatment, to define the role of $\mathrm{Cu}$ and $\mathrm{K}$ during the pretreatment on activity and selectivity and to define the chemical 
and physical changes which occur during the preferred pretreatment. The subtasks address each of these goals.

2.1 Determine the Role of $\mathrm{Cu}$ in the Activation of Precipitated Low- and HighAlpha, Iron-Based Fischer-Tropsch Catalysts.

2.2 Determine the Effect of $\mathrm{K}$ Content on Activation Procedures and Determine if the Method of Addition has any Effect on Catalyst Activity and Life.

2.3 Determine the Physical and Chemical Changes that Occur during Catalyst Pretreatment and Use and Determine how these Changes Effect the Strength of the Catalysts.

2.4 Evaluate the Effect of Carbon Deposition during Catalyst Activation on Activity, Selectivity and Aging Characteristics.

\section{c. Task 3.0 Catalyst Structure and Characterization.}

The goal of this task is to provide basic analyses (surface area, XRD) of all catalyst prepared and to provide additional techniques as required (Mössbauer, SEM, XPS, etc.) to answer specific questions or to provide basic required characterization data for the catalysts.

\section{d. Task 4.0 Catalyst Testing.}

The goals of this task are to operate the eight CSTR reactors, measure catalyst performance, determine the stable phases that exist during synthesis at low and high conversions and to determine the rates of interconversion of iron oxide and carbide.

4.1 Verify the Quality of Data Obtained from the CSTR's.

4.2 Measure Catalyst Performance. 
4.3 Determine the Stable Phases that Exist during Synthesis at Low and High CO Conversion Levels.

4.4 Obtain Data on the Rates Involved in the Interconversion of Iron Oxide and Iron Carbide.

\section{Results and Discussion}

Following the nomenclature of Sasol, we shall refer to low temperature and high temperature operational modes. As practiced at Sasol, the high temperature mode produces lower boiling products than the low temperature synthesis. Thus, the high temperature operation may be viewed as a low-alpha operation whereas the low temperature operation may be viewed as a high-alpha operation. The current production capacities at the Sasol plants are summarized below according to reactor and temperatures modes (1):

\begin{tabular}{||c|c|c|c|c||}
\hline Capacities & \multicolumn{5}{|c||}{ Bbl/Day } \\
\hline & CFB & SAS & TFB & SP \\
\hline Total installed cap & 110,000 & 11,000 & 3,200 & 2,500 \\
\hline Capacity/reactor & 6,500 & 11,000 & $500-700$ & 2,500 \\
\hline \multicolumn{7}{|l||}{ Potential/reactor } & 7,500 & 20,000 & 1,550 & 10,000 \\
\hline $\begin{array}{l}\text { CFB }=\text { Circulating Fixed Bed } \\
\text { SAS = Sasol Advanced Synthol Fixed Fluid Bed } \\
\text { TFB = Tubular Fixed Bed } \\
\text { SP = Slurry Phase }\end{array}$ &
\end{tabular}

The product selectivities on a carbon basis for these two operational modes are: 


\begin{tabular}{||l|c|c||}
\hline \multicolumn{1}{|c|}{ PRODUCT } & LTFT & HTFT \\
\hline Methane & 4 & 7 \\
\hline $\mathrm{C}_{2}-\mathrm{C}_{4}$ olefins and paraffins & $8(50 \%)^{\mathrm{a}}$ & $30(80 \%)^{\mathrm{a}}$ \\
\hline Gasoline & 18 & 36 \\
\hline Middle distillate & 19 & 12 \\
\hline Heavy oils and waxes & 48 & 9 \\
\hline Water soluble oxygenates & 3 & 6 \\
\hline a. Number in parenthesis is the carbon \% olefins. \\
\hline
\end{tabular}

Both of these distributions are fit by a "two-alpha" plot corresponding to synthesis by chain growth to produce a low molecular weight and a high molecular weight products. Based on the Sasol data for the low-temperature operation, it is calculated that about 50 carbon \% is produced by each synthesis mode; however, for the high temperature mode $75 \%$ of the products are produced by the low-alpha synthesis pathway. As stated above, the high-temperature mode (low alpha mode) accounts for more than $95 \%$ of the total installed capacity at the Sasol commercial plants.

During about 40 years of operation Sasol has made significant advances in their reactor technology. Thus, the older-type fixed bed reactors (Arge) have been replaced by a slurry-phase reactor; the slurry reactor has been operated for more than 2 years without problems and at, or above, the design capacity. One Sasol Advanced Synthol (SAS) fixed-fluidized bed reactor has been installed to replace Synthol-circulating fluidized bed (CFB) reactors and sufficient SAS reactors have been ordered to replace all of the remaining older CFB reactors.

The capacity of the high-temperature, low-alpha CFB and SAS units compared to the low-temperature, high-alpha units is overwhelming and corresponds to over $95 \%$ of the production capacity. The transportation fuel is split 60:40 gasoline:diesel in 
South Africa. Since most of the reactor-wax produced in the low-temperature process is refined to supply the world's demand for paraffin wax, the contribution by Sasol to the diesel fuel requirements for South Africa must come from their high-temperature, lowalpha operation.

Sasol plans to replace the 110,000 capacity currently supplied by the CFBs with SAS fixed-fluidized bed reactors, and not with the high-molecular weight, lowtemperature slurry reactors. Presumably Sasol is taking the view that the low-alpha, high-temperature synthesis will be an economical process for the next 20+ years. It should be obvious that Sasol considered both low- and high-temperature modes of operation before deciding to take the high-temperature route. Thus, Sasol's actions do not agree with the opinion of many that the only route available to produce transportation fuel is the low-temperature, heavy-product route.

It does not appear to be possible to operate with a cobalt or ruthenium catalyst in a mode other than one leading to high molecular weight products (high alpha mode). However, even for the cobalt catalyst the published data show the two-alpha product distribution. This means that $50 \%$, and usually greater than $50 \%$, of the products from the Co or Ru catalyst are too heavy to be utilized directly as transportation fuels. Thus, a second process step (hydrocracking) must be utilized to convert these high molecular weight products to transportation fuels.

In order to develop a process based on the cobalt catalyst, Shell has opted to utilized a fixed-bed reactor F.-T. process. Shell acquired the Gulf-Badger catalyst process technology from Chevron. The Shell fixed-bed operation in Malaysia utilizes a reactor system that is virtually identical to the one utilized by Sasol for their ARGE reactors that utilized an iron catalyst and that are now being replaced by slurry reactor 
technology. The Exxon AGC-21 process utilizes a proprietary catalyst for both the F.-T. (presumably based on cobalt) and hydrocracking steps that are included in their process.

\subsection{Iron Catalyst Preparation}

Considering the vast literature back through the German work prior to and during WWII, it appears that the preferred iron catalyst is obtained through a precipitation procedure. The most economical source of iron in the U.S. is a concentrated solution of ferrous sulfate that is produced as a by-product in the manufacture of iron and/or steel. However, it is virtually impossible to remove all of the sulfate that is incorporated during the precipitation step even by repeated washing. Thus, in spite of the greater cost of iron, ferric nitrate appears to be the preferred source of iron for the preparation of iron Fischer-Tropsch catalysts.

\subsection{1. $\underline{\text { Sasol }}$}

Catalyst robustness is a problem. Sasol utilizes a precipitation step for the preparation of the catalyst that is utilized in the fixed-bed ARGE and the slurry reactor (2). The same procedure is used to add the chemical and structural addition step that follows the precipitation and washing steps. Further processing of the fixed-bed ARGE catalyst involves filtering the slurry, extruding the "paste" and then drying the extrudate. For the preparation of the slurry-phase catalyst, the catalyst slurry is spray dried; the dried particles of the desired size are separated from fines and/or oversized particles using a cyclone/screening/scribbing system. It is found that the breakage of the spherical catalyst particles formed for the slurry reactor is inversely proportional to the mechanical strength of the particle. However, it is reported that the formation of ultra fine particles due to abrasion does not necessarily follow this trend [of 
abrasion/mechanical strength]. This implies that the measurement of the mechanical strength of the oxide catalyst precursor does not have predictive value for catalyst integrity during use in a slurry reactor.

Sasol provides pictures representing a batch of good catalyst particles and bad catalyst particles (Figure 1). The good particles are spherically shaped and do not exhibit imperfections. The poor catalysts exhibit indentations that extend into the body of the pellet and is indicative of an operation that involves non-uniform drying during the spray-drying process. The catalysts spray-dried at UCI for the La Porte runs exhibit the characteristics of the good catalysts described by Sasol (Figure 2) (3). Furthermore, the attrition of the carbide form of the good spheres prepared by $\mathrm{UCl}$ show a gradual decrease in size during tumbling experiments under non-reacting conditions (3).

\subsubsection{Ruhrchemie}

Kuntze, et al. (4) tested a precipitated iron catalyst that they report to be similar to one used in the ARGE-process, at least during the early work at Sasol. The composition of the catalyst was (mass units): $\mathrm{Fe}: \mathrm{SiO}_{2}: \mathrm{Cu}: \mathrm{K}_{2} \mathrm{O}=100: 25: 5: 5$. When this catalyst was reduced in hydrogen at $220^{\circ} \mathrm{C}$, it was reported to have a reduction degree of $32 \%$ (not defined as to whether this means to $\mathrm{Fe}_{3} \mathrm{O}_{4}$ or to $\mathrm{Fe}$; similar catalysts at CAER would be reduced to $\mathrm{Fe}_{3} \mathrm{O}_{4}$ under these conditions) and had a surface area of $235 \mathrm{~m}^{2} / \mathrm{g}$.

\subsubsection{The "Reichsamt" Comparative Experiments}

This test utilized six different catalysts representing the major German developers of the F.-T. process at that time (WWII period) (5). These tests were under the supervision of Herbet Kölbel. The tests were conducted at temperatures not to 
exceed $225^{\circ} \mathrm{C}, 10 \mathrm{~atm}$. pressure, without recycle, and were to last for three months without change of catalyst. The products that were produced had to resemble sufficiently those obtained with cobalt catalysts so as to be marketable as substitutes for the cobalt-produced products. The catalyst compositions are summarized below:

\begin{tabular}{|c|c|}
\hline ORGANIZATION & CATALYST COMPOSITION \\
\hline $\mathrm{KWI}$ & $\mathrm{Fe}, 100: \mathrm{Cu}, 1: \mathrm{K}_{2} \mathrm{CO}_{3}, 1$ \\
\hline Lurgi & $\mathrm{Fe}, 100: \mathrm{Cu}, 1.0: \mathrm{SiO}_{2}, 30: \mathrm{K}_{2} \mathrm{O}, 2.0$ \\
\hline Brabag & $\mathrm{Fe}, 100: \mathrm{Cu}, 2 \cdot 0, \mathrm{Zn}, 20: \mathrm{K}_{2} \mathrm{CO}_{3}, 1$ \\
\hline IG & Sintered iron containing $\mathrm{Al}_{2} \mathrm{O}_{3}$ and $\mathrm{K}_{2} \mathrm{O}$ and $\mathrm{CaF}$ as support \\
\hline Rührchemie & $\mathrm{Fe}, 100: \mathrm{Cu}, 5: \mathrm{CeO}_{2}, 10: \mathrm{kieselguhr,50}$ \\
\hline Rheinpreussen & $\mathrm{Fe}, 100: \mathrm{Cu}, 5: \mathrm{CaCO}_{3}, 100: \mathrm{K}_{2} \mathrm{CO}_{3}, 0.5-1.0$ \\
\hline
\end{tabular}

The above catalysts were tested in a 5 -liter reactor and were presumably sufficiently robust to be utilized in a fixed-bed reactor. In general, there were only minor differences in the activity and limited product selectivities that were reported.

Sasol also uses a precipitation procedure that was described above.

\subsection{4. $\underline{\text { Mobil }}$}

These workers utilized a precipitated iron catalyst that contained copper and potassium. Several formulations were utilized but the compositions appear to be considered proprietary and were not provided in their DOE report. In one run, the Mobil workers changed from a low-alpha operation to a high-alpha operation merely by adding a soluble organic potassium salt at a point during the run; thus, they utilized the same base catalyst for both the low- and high-alpha in at least one of their runs. Based on a Mobil patent for an iron catalyst, we speculate that the Mobil low-alpha catalyst resembled the catalyst used in La Porte run II (except for the Cu level which was in the $2 \%$ range in the Mobil patent and was $12 \%$ in the La Porte run). 


\subsubsection{Rentech}

The catalyst preferred by Rentech was an unsupported precipitated iron catalyst promoted with copper and potassium (6). Metallic iron and copper were dissolved in nitric acid to form a mixture of ferrous/ferric iron and precipitation was effected by adding ammonium hydroxide to produce a $\mathrm{pH}$ of 7.4 . The precipitate is washed free of ammonium nitrate. A slurry containing the proper amount of potassium carbonate was then spray dried to produce particles with diameters in the range of 5 to 50 microns. The final step in catalyst preparation was heating in a fluidized bed at $600^{\circ} \mathrm{F}\left(315^{\circ} \mathrm{C}\right)$.

\subsubsection{China}

These workers used a continuous precipitation procedure to produce their catalysts (7). They report that both low and high $\mathrm{pH}$ conditions are to be avoided in order to obtain a high surface together with the preferred pore size distribution.

\subsection{Iron Catalyst Activation}

\subsubsection{The "Reichsamt" Comparative Experiments}

A variety of activation procedures were utilized in this test (5). Presumably these were specified by the organization that provided the catalyst and represented the preferred procedure for each catalyst formulation. For the KWI catalyst listed above the activation consisted of reduction in synthesis gas $\left(\mathrm{H}_{2} / \mathrm{CO}=2\right)$ at $325^{\circ} \mathrm{C}$ and $0.1 \mathrm{~atm}$. (absolute) for 24 hours. The Lurgi catalyst was reduced in hydrogen at about $250^{\circ} \mathrm{C}$. The Brabag catalyst was activated by treatment in water gas at $235-240^{\circ} \mathrm{C}$ for 48 hours and the Rheinpreussen catalyst received a similar activation at atmospheric pressure. The Rührchemie catalyst was reduced in hydrogen at $300^{\circ} \mathrm{C}$. The catalyst preparation and activation procedures are summarized in the following table:

Table 1 


\section{Summary of the Catalyst Preparation and Pretreatment Procedures}

Company

Ruhrchemie

Kaiser Wilhelm

Inst., Mülheim)
Catalyst Preparation

Conventional precipitation with

$\mathrm{Fe}, 100: \mathrm{Cu}, 5: \mathrm{CaO}, 10:$ kieselguhr, 150

Precipitated from nitrate solution

$\mathrm{Fe}, 100: \mathrm{Cu}, 1-3: \mathrm{K}_{2} \mathrm{CO}_{3}, 0.1-1.0$
Pretreatment

Hydrogen reduction, $1 \mathrm{hr}$., at $300^{\circ} \mathrm{C}$; or recycle gas.

$\mathrm{CO}$ at $325^{\circ} \mathrm{C}, 0.1$ atm.; $\mathrm{Fe}_{3} \mathrm{C}$ formed, claimed as active phase.

Hydrogen reduction at 800 $850^{\circ} \mathrm{C}$
I.G. Farbenindestrie

AG (foam process)

I.G. Farbenindestrie AG (tubular react.)

I.G. Farbenindestrie AG (Synol process)

Lurgie Gesellschaft für Wärmetechnik

Rheinpreussen
Paste of iron powder (from decomp. of $\mathrm{Fe}(\mathrm{CO})_{5}, 1 \%$ borax, sinter $800-900^{\circ} \mathrm{C}$.

Paste of iron powder (from decomp. Hydrogen reduction at of $\mathrm{Fe}(\mathrm{CO})_{5}$ with $\mathrm{K}_{2} \mathrm{CO}_{3}$ or borate. $350-400^{\circ} \mathrm{C}$.

Paste of iron oxide powder with 5-25\% Hydrogen reduction at $5-25 \% \mathrm{MgO}$ or $\mathrm{MgCO}_{3}$ and $1-2 \% 350-450^{\circ} \mathrm{C}$.

$\mathrm{K}_{2} \mathrm{CO}_{3}$ or borate, sinter at

$850^{\circ} \mathrm{C}$ in nitrogen.

Melting iron in oxygen incorporating $2 \%$ alumina and $1 \% \mathrm{~K}_{2} \mathrm{O}$

Precipitated from nitrate solution with with sodium carbonate at boiling point, wash and add $\mathrm{K}_{2} \mathrm{CO}_{3}$. $\mathrm{Fe}, 100: \mathrm{Cu}, 2.5$ :alumina, $9: \mathrm{K}_{2} \mathrm{O}$, 2:kieselguhr,30.

Precipitated catalyst with $\mathrm{Fe}, 100$ : $\mathrm{Cu}, 1.0$ :kieselguhr, $50: \mathrm{K}_{2} \mathrm{CO}_{3}, 2$.
Hydrogen reduction at $450^{\circ} \mathrm{C}$.

Hydrogen reduction at $250-350^{\circ} \mathrm{C}, 1 \mathrm{hr}$.
$\mathrm{CO}$ at low partial pressure.

\subsubsection{Rentech}

These workers (6) report that "Determining the 'best' activating procedure for a catalyst is difficult at best even if it is known what changes in the catalyst are needed to give the desired activity, selectivity and stability." They utilized a complex recipe, initially starting by heating in an inert gas, then switching to synthesis gas at a temperature higher than the synthesis temperature using a hydrogen-rich synthesis 
gas. The inventors indicate that "It is believed that the presence of this water [from the activation] prevents over-carburization of the catalyst and thereby improves the activity and selectivity of the catalyst." and quote Koenig et al. as support for this view (8).

\subsubsection{China}

These workers made a study of the effect of activation on the catalytic properties of their iron catalyst (7). They report that reduction of the catalyst with hydrogen leads to the production of Fe and a catalyst with a low surface area and low catalytic activity. They conclude that reduction in hydrogen is not a preferred activation procedure. They also studied the use of syngas mixtures in which the $\mathrm{H}_{2} / \mathrm{CO}$ ratio was varied from 1 to 9 . The more active catalyst appeared to be produced following activation with the lower $\mathrm{H}_{2} / \mathrm{CO}$ synthesis gas at the lower activation pressure (0.3-1.0 MPa). However, they preferred a staged activation in which the treatment with syngas is carried out in steps of increasing temperature.

\subsection{4. $\underline{\text { CAER }}$}

Extensive pretreatment studies have been conducted at the CAER with a variety of iron catalysts. Initially, the catalyst activation approach followed a staged heat-up in syngas $\left(\mathrm{H}_{2} / \mathrm{CO}=0.7\right)$ to finally attain a temperature of about $20^{\circ} \mathrm{C}$ higher than the reaction temperature $\left(270^{\circ} \mathrm{C}\right)$. Presumable because of some residual sulfur in the catalysts prepared to by UCI to be screened for La Porte Run II, sufficient sulfur accumulated on the surface so that the catalytic activity was low following this pretreatment procedure. The same catalysts could be activated successfully in pure CO, either at atmospheric or reaction (170 psig) pressure.

Using pure CO, CAER workers found that they could obtain an active material following 24 hours in $\mathrm{CO}$ at $270^{\circ} \mathrm{C}$. Four activation runs were made at the CAER in the 
2 inch diameter $\times 6$ foot long using the spray-dried Round-Robin batch of unsupported iron catalyst of the same formulation used for La Porte Run II. These runs were made to produce activated (carbided) catalyst samples to be used in filtration studies at Mott. The first resulted in a "poor" run due to the use of a low CO flow rate; the other three runs were at a lower pressure and provide reproducible data (Figure 3). These runs were conducted until the catalyst had accumulated an amount of carbon, based upon the cumulative production of $\mathrm{CO}_{2}$ measured in the exit gas, to produce about $10 \%$ more carbon that required to produce $\mathrm{Fe}_{2.2} \mathrm{C}$. The data generated in the CSTR studies at the CAER had the same shape as those obtained in the $2 " x 6$ ' slurry reactor except that it took 17, rather than 18 hours, to attain the desired amount of carbon deposition. The activation at LaPorte for Run II used a similar activation procedure (40 wt.\% slurry) and produced data that followed closely the "poor" curve in Figure 4.

Synthesis gas and pure hydrogen streams are, or can be, easily attained at a commercial F.-T. site. However, it is not easy to obtain a pure stream of $\mathrm{CO}$ at a commercial F.-T. site. Thus, while CO pretreatment may be of interest for laboratory studies or for use at La Porte, it does not appear to be easily practiced at a commercial site.

Activation in $\mathrm{CO}$ occurs in steps. Initially, there is a rapid reaction to convert the $\mathrm{Fe}_{2} \mathrm{O}_{3}$ to $\mathrm{Fe}_{3} \mathrm{O}_{4}$; the formation of $\mathrm{Fe}_{3} \mathrm{O}_{4}$ has been confirmed by Mössbauer spectroscopy. After the formation of $\mathrm{Fe}_{3} \mathrm{O}_{4}$ is complete, a slower reaction occurs to deposit carbon in the form of an iron carbide (and possibly carbon to coat the iron carbide particle).

Activation in synthesis gas at reaction pressure appears to be dependent upon the catalyst formulation. For instance, the catalyst that Ruhrchemie provided to DOE 
could be successfully activated in syngas at reaction pressure. A catalyst with a similar composition that was prepared at the $\mathrm{UCl}$ could not be successfully activated using syngas. It appears that it is essential to have copper (or some metal that functions in a similar manner) present if the catalyst is to be activated in synthesis gas at or near reaction pressure. Even when copper is present, it appears that the approach to maximum activity requires days of operation in the synthesis mode.

The effectiveness of an activation at reaction pressure using gas flow rates at or near to those to be used for the synthesis depends upon the hydrogen partial pressure. Thus, an active catalyst was obtained when pure CO was passed over the catalyst for 24 hours prior to changing to the synthesis conditions (Figure 5). However, there appears to be a linear decline in activity as the partial pressure of hydrogen in the feed gas is increased.

On the other hand, activation with synthesis gas was easily accomplished at atmospheric pressure during 24 hours at reaction temperature. This observation is in agreement with much of the early German work on activation of iron catalysts.

Reduction in hydrogen is a complex operation. Initially $\mathrm{Fe}_{3} \mathrm{O}_{4}$ is formed; this stage is followed by the reduction to metallic iron at a higher temperature and/or longer reaction time. During a 24 hour period at $270^{\circ} \mathrm{C}$ it is found that about $30 \%$ of the iron is present in the metallic form and the remaining iron is present as $\mathrm{Fe}_{3} \mathrm{O}_{4}$. When synthesis gas contacts the reduced catalyst at reaction temperature, within 2 hours or less the metallic iron is converted to iron carbides. The presence of copper has been shown to result in a lowering by $20-40^{\circ} \mathrm{C}$ the temperature where each of these two reductions occur. In general, reduction in hydrogen prior to contact of the catalyst by synthesis gas can produce an active catalyst, and this appears to be the procedure that 
is utilized at Sasol in their commercial operation. On the other hand, it does not appear that the optimum degree of conversion to metallic iron has been reported in the open literature. It has been reported that complete reduction to metallic iron is to be avoided since the metallic iron sinters rapidly at this temperature to produce a low area material that results in a low-activity catalyst.

\subsection{Cobalt Catalysts}

The catalysts utilized in the German commercial plants prior to and during WWII was primarily based upon cobalt and these were operated at atmospheric or low pressure conditions. Roelen encountered severe loss in activity during the use of the cobalt catalyst in the early plants but overcame these problems, primarily by separating the interfering elements from the cobalt by precipitation prior to catalyst preparation (9).

During the past 30 years, a vast amount of patent and open literature has developed on cobalt catalyst; these are primarily devoted to the use of one or more elements to modify the catalytic properties of the cobalt.

During the 1970s, Gulf Oil workers found that a Group VIII element, such as ruthenium $(\mathrm{Ru})$, incorporated in a much smaller amount compared to cobalt $(\mathrm{Co})$, greatly increased the activity of the finished catalyst; as an example, a catalyst containing 20 wt.\% Co would contain 1 wt.\% Ru (10). In addition to cobalt and the Group VIII metal, other components, such as magnesia and thoria (Shell now uses zirconia) were incorporated in order to improve the performance of the catalyst. A catalyst based upon the Gulf patent formulation became the basis for the Gulf-Badger process for the production of hydrocarbons using the Fischer-Tropsch synthesis. Two designs were tested - fixed-bed and fluidized-bed reactors - were tried. The operation of the fluidized-bed reactor was not satisfactory. Gulf Oil, working with Badger, 
designed, built and operated a 1 inch diameter, $40 \mathrm{ft}$. length (two-section) tubular reactor using the Gulf cobalt catalyst. The aged catalyst was reported to be restored to its original activity following an oxidation and reduction cycle.

Gulf Oil workers also patented the use of two stage operation with the first stage employing a Fischer-Tropsch catalyst and the second stage employing an acidic catalyst such as the silicalite catalyst developed by Union Carbide. Alternatively, both the F.-T. and the acidic catalyst could be added to a single reactor. The utility of this concept was adequately demonstrated by the Mobil work carried out under DOE contract.

Unsupported cobalt catalysts have not been found to be satisfactory. A wide range of supports have been utilized; these include alumina, silica, titania, zirconia, magnesia, silica alumina, carbon, and molecular sieves. Recently, Statoil has been issued patents in which it is claimed that the use of alumina, in contrast to other supports, leads to the catalyst with a superior activity compared to Co on other supports (11). The Statoil work also indicates that the incorporation of alkali decreases the catalytic activity and increases the alpha value from about 0.7 for the cobalt only catalyst to 0.9 or greater as the $\mathrm{K} / \mathrm{Co}$ ratio increases. However, the conclusion for the alteration of the alpha-value by cobalt, from a scientific viewpoint if not from a patent viewpoint, is tenuous at best.

Exxon workers contend that the activity of all cobalt catalysts, with the exception of a titania supported cobalt, exhibits the same activity (site time yield) (Figure 6) (12). Thus, it appears that the Statoil and the Exxon data may be in conflict provided one makes the comparison upon the cobalt dispersion. In the Exxon view, the preferred catalyst involves placing cobalt on the external portion of the support ("egg-shell" type) 
to improve the selectivity towards the $\mathrm{C}_{5_{+}}$fraction. Exxon workers have provided extensive published work to indicate that, because of olefin reincorporation, a balance between the kinetics and the diffusional factors must be taken into account. Thus, a structural parameter ( $X$ in Figure 7), dependent upon the pellet diameter, the average pore size of the support, and the density of the metal sites within the pellet, acts to determine the F.-T. product distribution for supported Ru and Co catalysts. Thus, the proper control of the parameter $\mathrm{x}$ allows one to maximize the production of $\mathrm{C}_{5_{+}}$products and, at the same time, minimize the amount of undesirable methane.

There are many patents that purport to provide recipes to prepare cobalt catalysts in such a manner that a superior catalysts is produced with respect to activity, selectivity, and/or ability to handle the exothermic reaction. Unlike the case of the Exxon open literature reports on scientific aspects of the catalyst impact upon the F.-T. synthesis, little exists in the open literature to evaluate the various claims of these patents. It appears that legal actions are underway to resolve some of the perceived or real differences among some of the patents.

For cobalt catalyst, those containing magnesia had been in use in Germany since 1938. The introduction of the magnesia catalysts were claimed to have made possible catalyst lives of up to eight months at normal pressure. According to the managing director of Ruhrchemie A.-G. the magnesia was added solely to increase the hardness of the resulting catalyst and thus to reduce its tendency to disintegrate to dust in the reactor (13).

\subsection{Comparison of Cobalt and Iron Catalysts}

A major difference between cobalt and iron catalysts is the ability of iron to catalyze the water-gas-shift (WGS) reaction: 


$$
\mathrm{CO}+\mathrm{H}_{2} \mathrm{O} \rightleftharpoons \mathrm{CO}_{2}+\mathrm{H}_{2} .
$$

At Fischer-Tropsch synthesis conditions, the equilibrium for the above WGS reaction lies far to the right; that is, WGS is a thermodynamically favored reaction. Furthermore, the reverse reaction is slow, compared to the forward reaction, under Fischer-Tropsch conditions. However, the forward reaction depends upon the partial pressure of water.

For the F.-T. reaction, cobalt produces hydrocarbons and water whereas the iron catalyst, in the extreme condition, will produce hydrocarbons and carbon dioxide. Thus, for each $\mathrm{CH}_{2}$ produced as hydrocarbon, cobalt will produce $\mathrm{H}_{2} \mathrm{O}$ whereas iron will produce $\mathrm{CO}_{2}$. In this instance, iron will consume two $\mathrm{CO}$ molecules for each $\mathrm{CH}_{2}$ formed whereas the cobalt catalyst will consume only one $\mathrm{CO}$ for each $\mathrm{CH}_{2}$ formed. Thus, the "conventional wisdom" is that cobalt is the preferred catalyst since it makes more efficient use of the $\mathrm{CO}$ in the syngas.

For a coal derived syngas, this "conventional wisdom" is an illusion. To produce $\mathrm{CH}_{2}$ and $\mathrm{H}_{2} \mathrm{O}$ by the F.-T. reaction two molecules of $\mathrm{H}_{2}$ are required as shown in the following equation:

$$
\mathrm{CO}+2 \mathrm{H}_{2} \rightarrow-\mathrm{CH}_{2}^{-}+\mathrm{H}_{2} \mathrm{O} .
$$

Thus, using a ratio of $\mathrm{H}_{2} / \mathrm{CO}=0.7$ (the "middle-ground value" for a syngas produced from coal), there is a deficiency of hydrogen when the cobalt catalyst is used. The above F.-T. reaction can only take place provided an additional amount of $\mathrm{CO}$ is converted to $\mathrm{CO}_{2}$ and $\mathrm{H}_{2}$ to provide a synthesis gas to pass over the cobalt catalyst that has a ratio $\mathrm{H}_{2} / \mathrm{CO}=2$. Thus, with a syngas derived from coal the only difference between the use of the two catalysts is that with the iron catalyst the WGS reaction can be carried out in the same reactor as the F.-T. reaction whereas with the cobalt catalyst the WGS reaction must be carried out in a process operation that precedes the F.-T. 
reactor. The preferred catalyst, on the basis of the usage of syngas to produce hydrocarbons, will depend only on the economics of whether it is preferable to conduct the WGS reaction in the F.-T. reactor or in a separate operation.

With a syngas derived form natural gas, the $\mathrm{H}_{2} / \mathrm{CO}$ ratio is 2 so that "conventional wisdom" teaches that cobalt, without WGS activity, is definitely the preferred catalysts. This assertion is both "true" and "false." At high CO conversions, the WGS reaction is an important component of the synthesis using an iron catalyst and hydrogen is formed in excess of that needed to produce $\mathrm{CH}_{2}$. Thus, the "conventional wisdom" assertion for iron catalysts is "true" at high CO conversion levels. However, the extent of WGS reaction relative to the F.-T. reaction is very dependent upon the $\mathrm{CO}$ conversion level for an iron catalyst. Thus, the fraction of $\mathrm{CO}$ converted by the WGS reaction is low at low CO conversions (Figure 8). Thus, up to CO conversion of about $60 \%$, the WGS reaction does not make a significant contribution to the overall reaction of $\mathrm{CO}$. Thus, below about $60 \% \mathrm{CO}$ conversion iron and cobalt catalysts produce essentially the same products and therefore the same relative utilization of hydrogen and carbon monoxide. Therefore, it is only true that a cobalt catalyst is preferred for an operation that derives the syngas from natural gas when the iron catalyst is to be utilized in a process configuration that requires high $\mathrm{CO}$ conversion in a single-pass operation. Thus, the "conventional wisdom" assertion to favor cobalt over iron catalyst is "false" for operations with an iron catalyst involving recycle or multiple reactors so that $\mathrm{CO}$ conversion is kept at about $60 \%$ or less.

Thus, iron and cobalt catalysts have a similar selectivity for $\mathrm{CO}$ conversions at the $60 \%$ or lower levels. 
The other "conventional wisdom" viewpoint that favors the cobalt catalyst over the iron catalyst is that the cobalt catalyst is more active. Again, this "conventional wisdom" is both "true" and "false." The CO conversion for a cobalt catalyst is considered to depend only upon the dispersion of the cobalt as shown in Figure 9. This is not the case with an iron catalyst. As shown in Figure 10, the rate of F.-T. synthesis with an iron catalyst increases nearly linearly with time up to a conversion level of about $60 \%$ and then to increase only slowly with time above this level. Furthermore, the usage of hydrogen and carbon monoxide varies with $\mathrm{CO}$ conversion. Thus, if we compare cobalt and iron catalysts at high CO conversion levels the "conventional wisdom" that Co is more active catalyst than iron is "true". However, if we compare the two catalysts at temperatures where the production of $\mathrm{CH}_{4}$ is low (e.g., $220^{\circ} \mathrm{C}$ for cobalt and $270^{\circ} \mathrm{C}$ for iron), the grams of hydrocarbon produced per gram of catalyst is comparable. Thus, based on an operation using iron catalysts at $60 \%$ conversion and using recycle or multiple reactors to a attain the high overall conversion of syngas, the "conventional wisdom" is "false".

The above is based upon an analysis of the situation by CAER. Similar conclusions have been reached by Sasol investigators (14). Based upon syngas conversion, they show that using an iron catalyst at $240^{\circ} \mathrm{C}$ at high relative space velocities, the iron catalyst matches or even exceeds the activity of the cobalt; only at short relative space velocities (high conversion levels) does the conversion obtained with a cobalt catalyst exceed that of the iron catalyst. Furthermore, on the basis of a comparison of the two catalysts, the cobalt catalyst is more productive than an iron catalyst only at the lower relative space velocities and lower pressures (Figure 11). Also, as the operating temperature for the iron catalyst is increased, the dividing line in 
Figure 12 will lower so as to favor iron over cobalt to even higher conversions. Presumably the data in Figure 12 is for an iron catalyst designed to produce higher molecular weight products (high-alpha catalyst); thus, the use of an iron catalyst to produce low molecular weight products (low-alpha catalyst) would lower the dividing line to favor the iron catalyst by an additional amount. The Sasol workers report that the activity of their cobalt catalyst compares favorably with patented alternative cobalt catalysts. The above comparison was at an $\mathrm{H}_{2} / \mathrm{CO}=2$ ratio; i.e., a syngas that would be produced from natural gas.

The Sasol workers provide stability data for their cobalt catalyst operated at $220^{\circ} \mathrm{C}, 20$ bar (essentially $20 \mathrm{~atm}, 290 \mathrm{psi}$ ) and 0.5 relative space velocity. The authors state that a commercial syngas feed was used that contained $\mathrm{H}_{2} / \mathrm{CO}=2$ and contained 25 vol.\% inerts. Under these conditions there was a rapid decline in activity during about 5 days and then a very slow decline in activity during the next 20 days. These authors attribute the initial decline with build-up of waxes in the catalyst pores and the slow decline as probably due to a low level of sulfur poisoning.

Based upon the knowledge in the open and patent literature, it does not appear valid to make a choice between a cobalt and an iron catalyst unless the choice is based upon a particular process in which the operational conditions are specified.

\subsection{Slurry Reactor Studies}

\subsubsection{German}

\subsubsection{Roelen}

In spite of studies directed toward industrial development, the KWI pilot plant studies showed that the optimal stoichiometric ratio of $\mathrm{CO}: \mathrm{H}_{2}$ was $1: 2(9)$. In November 1930 the pilot plant staff attempted to run the F.-T. in the liquid phase, 
and they were successful in maintaining the heat flow problem. However, the typical catalysts of that day had an activity that was too low to have practical commercial interest.

\subsubsection{Kölbel}

Until the start-up of the slurry reactor by Sasol, the Rheinpreussen-Koppers demonstration plant was the largest slurry reactor that had been operated successfully. The reactor was $1.55 \mathrm{~m}$ in diameter and $8.6 \mathrm{~m}$ in height. Kölbel states that at the time that most work was conducted using the demonstration plant (1952-53), the operation was confined almost exclusively to the production of gasoline (15). The results of the operation of this plant and the smaller laboratory scale slurry phase reactor produced data that have become the "standard" that is used to compare with other slurry phase studies. A typical catalyst used by Kölbel would have a composition of $\mathrm{Fe}: \mathrm{Cu}: \mathrm{K}_{2} \mathrm{O}=$ 100:0.1:0.05-0.5; thus, the catalyst used by Kölbel would be consistent with the objective of producing gasoline range material, and not high molecular weight reactorwax. The catalyst used by Kölbel would be similar to a low-alpha iron catalyst described in a Mobil patent and the one used for La Porte run II (low, not the actual high Cu content).

\begin{tabular}{||l|c|c||}
\hline \multicolumn{3}{||c||}{ Table 2 } \\
\begin{tabular}{|l|c||} 
Operating Data and Results of Liquid-Phase Synthesis for One-Step Operation with \\
a Single Passage of the Gas Over Iron Catalysts (from ref. 15)
\end{tabular} \\
\hline \multicolumn{1}{|l|}{$\begin{array}{c}\text { Demonstration } \\
\text { Plant (a) }\end{array}$} & $\begin{array}{c}\text { Laboratory } \\
\text { Plant (b) }\end{array}$ \\
\hline $\begin{array}{l}\text { Effective reaction space (volume suspension } \\
\text { including dispersed gas) (L) }\end{array}$ & 10,000 & 6 \\
\hline Catalyst (kg Fe) & 800 & 0.4 \\
\hline Synthesis gas pressure (bar) & 12 & 11 \\
\hline Synthesis gas (volume ratio, $\left.\mathrm{CO}: \mathrm{H}_{2}\right)$ & 1.5 & 1.5 \\
\hline
\end{tabular}




\begin{tabular}{|c|c|c|}
\hline Quantity of synthesis gas $\left(\mathrm{Nm}^{3} / \mathrm{hr}\right)$ & 2,700 & 1.3 \\
\hline $\begin{array}{l}\text { Linear velocity of the compressed gases at operating } \\
\text { temperature referred to the free reactor cross section } \\
(\mathrm{cm} / \mathrm{sec})\end{array}$ & 9.5 & 3.5 \\
\hline $\begin{array}{l}\text { Total } \mathrm{CO}+\mathrm{H}_{2} \text { used }\left(\mathrm{Nm}^{3} / \mathrm{hr}\right) \\
\text { Per m } \mathrm{m}^{3} \text { of reaction chamber }\left(\mathrm{Nm}^{3} / \mathrm{hr}\right) \\
\text { Per kg of } \mathrm{Fe}\left(\mathrm{Nm}^{3} / \mathrm{hr}\right) \\
\end{array}$ & $\begin{array}{l}2,300 \\
230 \\
2.6 \\
\end{array}$ & $\begin{array}{r}1.1 \\
183 \\
2.45 \\
\end{array}$ \\
\hline Average synthesis temperature, ${ }^{\circ} \mathrm{C}$ & 268 & 266 \\
\hline CO conversion, \% & 91 & 90 \\
\hline $\mathrm{CO}+\mathrm{H}_{2}$ conversion, $\%$ & 89 & 88 \\
\hline $\begin{array}{l}\text { Synthesis products referred to } \mathrm{CO}+\mathrm{H}_{2} \text { used: } \\
\qquad \begin{array}{cc}\text { Hydrocarbons } & \mathrm{C}_{1}^{+}\left(\mathrm{g} / \mathrm{Nm}^{3}\right) \\
& \mathrm{C}_{1}^{+}+\mathrm{C}_{3}\left(\mathrm{~g} / \mathrm{Nm}^{3}\right) \\
& \mathrm{C}_{3}^{+}\left(\mathrm{g} / \mathrm{Nm}^{3}\right) \\
\end{array}\end{array}$ & $\begin{array}{c}178 \\
12 \\
166 \\
\end{array}$ & $\begin{array}{c}176 \\
11 \\
165\end{array}$ \\
\hline O-containing products in the synthesis water $\left(\mathrm{g} / \mathrm{Nm}^{3}\right)$ & 3 & 2 \\
\hline $\begin{array}{l}\text { Space-time yield of } \mathrm{C}_{3}{ }^{+} \text {products including O-products } \\
\text { in } 24 \mathrm{hr}\left(\mathrm{kg} / \mathrm{m}^{3} \text { of reaction chamber) }\right.\end{array}$ & 930 & 740 \\
\hline
\end{tabular}

At the conversion level shown in the above table, only $178 \mathrm{~g}$ of hydrocarbons were produced per $\mathrm{m}^{3}$ gas (from the original paper in German, it was not possible for even a native German to tell whether this volume of gas refers to the amount of gas fed or to the amount of gas converted). Even if it is taken as the amount fed, at the $90 \%$ conversion level, more than $178 \mathrm{~g}$. of hydrocarbons should have been produced. For example, in the Mobil runs more than $200 \mathrm{~g}$ hydrocarbon were produced. Sasol workers indicate that they could not repeat Kölbel's results in their early studies (2). Kölbel et al. report that through polymerization of lower olefins, about $18 \mathrm{~g} / \mathrm{Nm}^{3} \mathrm{CO}+\mathrm{H}_{2}$ of alkylate gasoline can be produced. When this was mixed with the reformed gasoline $\left(112 \mathrm{~g} / \mathrm{Nm}^{3} \mathrm{CO}+\mathrm{H}_{2}\right), 130 \mathrm{~g} / \mathrm{Nm}^{3} \mathrm{CO}+\mathrm{H}_{2}$ of finished gasoline could be produced. For a CO conversion of $91 \%$, the $\mathrm{H}_{2}+\mathrm{CO}$ conversion was $89 \%$; the feed gas ratio was $\mathrm{H}_{2} / \mathrm{CO}$ $=0.67$. With this gas ratio the only way, based upon CAER results, that Kölbel could 
have obtained such similar high $\mathrm{CO}$ and $\mathrm{CO}+\mathrm{H}_{2}$ conversions would be to operate so that the single pass conversion was $50-60 \%$ and to recycle the unconverted gas. It has not been widely appreciated that much of the work that Kölbel reports has been conducted under conditions designed to produce gasoline; in this mode the demand on wax/catalyst separation is minimal. Thus, much of Kölbel's work can be viewed as being conducted under conditions that make the operation of a slurry reactor much easier than the current goal of operating to maximize the reactor-wax fraction to subsequently hydrocrack to produce diesel fuel.

Kölbel stressed that the low viscosity and surface tension of the liquid was crucial for maintaining the small bubble size needed to maintain gas-liquid mass transfer. Kölbel maintained the view that it was necessary to establish upper limits upon the solids content of the slurry in order to maintain a low viscosity.

\subsubsection{British}

The operation of the British plant was terminated about the time that they had solved most of the operating problems and considered themselves to be at a point where they could operate to produce reliable data. For example, low catalyst activity and rapid catalyst aging were problems than limited the usefulness of the data produced during the period of operation of the plant.

\subsubsection{U.S. Bureau of Mines}

The Bureau of Mines operated a 3 inch diameter $\times 8$ foot long reactor as well as a larger 8 inch diameter reactor in the oil-recycle mode. These units were operated with a precipitated and a fused iron catalyst that has a very low activity compared to the high surface area precipitated iron catalyst. The fused catalyst was used because it was hard and seemed to have the physical strength needed. Some experimental 
operating difficulties made it difficult to maintain constant temperature during significant portions of the runs. While it was demonstrated that this mode of operation was viable, little else was obtained that merit further consideration here.

\subsubsection{Mobil}

The initial runs in the pilot plant (Figures 13 and 14) at Mobil Oil, based upon the catalyst compositions in Mobil's patents, would utilize a catalyst with a composition that resembles the one reported above for Kölbel's work and the catalyst intended (low, not high, Cu content) for the La Porte Run II. Mobil's data from work funded by DOE have become the "standard" for both economic (e.g., 16) and technological evaluations (e.g., 17). The first three runs in the Mobil plant were conducted using a catalyst that produced low molecular weight materials; during the third run a potassium salt was added at 81 days-on-stream and this decreased the methane + ethane production from about 13 to 18 wt.\% without significantly lowering the CO conversion; however, operational upsets prevented a valid assessment of the impact of the added alkali (18). The later runs were in the high molecular weight product mode (wax mode). In most runs Mobil operated with about a 20 wt. $\%$ slurry catalyst loading. In run 8 , the aging rate of the iron catalyst operated at $250^{\circ} \mathrm{C}, 1.48 \mathrm{MPa}$ (about $15 \mathrm{~atm} ; 215 \mathrm{psi}$ ) and 1.4 $\mathrm{NL} / \mathrm{gFe}-\mathrm{hr}$ was such that half the activity would be lost during 24 days; later in the run the catalyst half-life was 13 days when the temperature and pressure were increased. Mobil workers indicate that the catalyst used in this run was not acceptable because of its high aging rate. In run 9, a surprisingly low methane + ethane make (about 5.4 wt.\%) was obtained. The catalyst was the "same" as had been used in a prior run where this was not observed; the only difference noted was that the low methane + ethane catalyst had a lower surface area. An operational upset terminated the effective 
operation at day 10 . In run 10 , Mobil workers reported that the catalyst could not be fully activated at synthesis conditions.

Run 12 was operated for 17 days at constant conditions and "This period represented the finest example of low methane + ethane [4.1 wt.\%] mode operation we have ever produced in the pilot plant." Wax production was about 60 wt.\%. An operational upset occurred on day 17 and afterwards catalyst settling and low catalyst activity were problems that could not be overcome.

Run 13 was a repeat of run 12 and good operation was accomplished for 35 days-on-stream, after which catalyst settling became a problem. Viewing the pictures of the catalyst, Mobil was utilizing particles in the 1-5 micron range, and the final catalyst particles were considerably larger following removal from the reactor. It is not clear whether this is due to catalyst particle growth or, more likely, cementing together several particles by reactor wax. It is not clear, if wax caused the particle size increase, whether this occurred in the reactor itself or was an artifact introduced during catalyst collection and subsequent treatment.

As stated above, the Mobil data have replaced the Kölbel data as the "standard" for slurry F.-T. operation. Data for reactor wax yield of 46 wt.\% are shown in Figure 15. This data has been utilized by Bechtel Corp. for their analysis of slurry F.-T. operations. They consider the data to consist of three regions: methane $\left(\alpha_{1}\right)$ that is higher than ASF; $\mathrm{C}_{2}-\mathrm{C}_{4}\left(\alpha_{2}\right)$ and reactor wax $\left(\alpha_{3}\right)$. Theoretical curves for reactor wax make of 9.49 wt. \% (low alpha data), 46.02 (intermediate alpha data) and 75.95 (high alpha data) are shown in Figure 16. 
Thus, the Mobil data, in spite of operational problems, represents the best data that is available in the open literature in sufficient detail that its quality can be adequately judged.

\subsection{5. $\underline{\text { Sasol }}$}

Sasol has reported general, but few specific, details about the development and operation of their slurry reactor operations. Sasol's work on a small scale began in the early 1980 s (2). In 1990, a slurry bed with a diameter of about $1 \mathrm{~m}$ was commissioned and the results confirmed their early expectations. In a bold move, Sasol decided to construct a commercial scale slurry reactor ( $5 \mathrm{~m}$ diameter, $22 \mathrm{~m}$ high) rather than two 5,000 tube tubular-fixed-bed reactors for the expansion of their low temperature operation. The commercial reactor was commissioned in May 1993 and has been reported to operate successfully since that time.

Sasol uses a separate catalyst pretreatment reactor in which hydrogen reduction (extent of reduction not specified) is used to activate a catalyst prior to its introduction into the slurry reactor. During operation, it is understood that an activated catalyst batch is on "stand-by" so that if a significant upset, such as a slug of sulfur to cause severe catalyst poisoning, causes a significant loss in productivity, the reactor is emptied and a fresh catalyst batch added during a short period. On-line catalyst removal and additions are reported to be done without difficulty. Based upon reports of the extent of sulfur poisoning in the fixed-bed ARGE reactors, it should not be surprising if Sasol operators had made several replacements of the catalyst inventory during the four years of commercial operation.

The authors (2) describe the churning nature of the slurry-base bubble interactions, implying that the Sasol operation operates in the bubbly, rather than slug, 
flow condition. Because of the isothermal nature of the slurry reactor, operating temperature can be much higher than in a fixed-bed tubular reactor without fear of hot spots leading to carbon formation and break-up of the catalyst. Hot-spots in the fixedbed reactor presumably allows for the catalyst in the hot-spot to reach a temperature sufficiently above that of the reactor set-temperature so that carbon formation becomes possible.

It is reported that for an iron catalyst, the product slate is considerable affected by the age of the catalyst, with wax selectivities decreasing with time. It is reported that "by proper scheduling of catalyst renewal, it is possible to maintain a steady selectivity profile for a single reactor while minimising the catalyst consumption." It therefore appears that the Sasol operation involves a regular schedule of catalyst addition, presumably to replace catalyst that is intentionally withdrawn as well as that which is lost as catalyst fines due to catalyst attrition.

Foam was found to build up in the reactor under certain conditions but it was reported that this could be prevented by modifying operation procedures. "Separation of gas from the entrained slurry was another development that was easily resolved.", implying that slurry carry-over can be a problem if not properly handled.

Several approaches were tried at Sasol in order to effect wax separation form the catalyst containing slurry. These included close attention to the production of the catalyst and its physical characteristics and to the separation processes. The technique currently in use in the commercial operation is considered to be proprietary information.

\subsubsection{China}


The Chinese have operated a two stage process involving slurry F.-T. synthesis with an iron catalyst and fixed-bed cracking/oligomerization processing using a ZSM-5 catalyst to convert the F.-T. product to gasoline range products (7). The F.-T. slurry reactor was $4 \mathrm{~cm}$ in diameter and $450 \mathrm{~cm}$ in height. The reactor, in a schematic form, is very similar to the one used by Mobil Oil (Figures 13,14). They used an unsupported precipitated iron catalyst with a typical composition of $\mathrm{Fe}: \mathrm{Cu}: \mathrm{K}_{2} \mathrm{O}=99.5: 0.5: 0.29$. The sample of catalyst used in the slurry reactor had obviously been calcined (based on CAER work, at temperatures of at least $300^{\circ} \mathrm{C}$ ) since the XRD analysis showed that the main crystal phase was $\alpha-\mathrm{Fe}_{2} \mathrm{O}_{3}$. The authors indicate that diffusion effects could be neglected for their runs. It appears that they used a slurry that contained $12 \%$ catalyst. Most of the published data concerning runs with their pilot plant are for the product following processing with the ZSM-5 catalyst. However, based upon data presumed to be for the F.-T. only operation, the liquid phase is reported to have a composition of approximately $70 \% \mathrm{C}_{5-12}, 27 \% \mathrm{C}_{13-22}$ and $3 \% \mathrm{C}_{22+}$. Thus, based upon the catalyst composition, the product distribution would be considered to originate from a low-alpha mode of operation, and the composition of the catalyst is consistent with this.

Furthermore, the low-alpha mode would probably be preferred for subsequent conversion of the F.-T. products with a ZSM-5 catalyst in the second stage.

The output during the course of a 1,000 hour (40 day) run declined due to loss of catalyst. From the published data on the Chinese F.-T. only operation, it is difficult to reach definitive conclusions or catalyst performance.

\subsection{Products}

\subsubsection{Low Temperature vs High Temperature}


The product distribution reported by Kölbel was typical of high temperature (low alpha) operation: $\mathrm{C}_{1-2}: \mathrm{C}_{3-4}:$ gasoline $\left(25-190^{\circ} \mathrm{C}\right)$ :diesel oil $\left(190-310^{\circ} \mathrm{C}\right)$ :heavies $\left(>310^{\circ} \mathrm{C}\right)$ $=7: 17: 62: 10: 3$ (19). The olefin content of the $C_{2-4}$, gasoline and diesel fractions were 72,74 , and $45 \%$, respectively. Kölbel reported in less detail on runs made to produce "medium" and "high" molecular weight products in addition to the ones described above. These products are shown in the following table:

\begin{tabular}{||c|c|c|c||}
\hline \multicolumn{4}{|c||}{ Table 3 } \\
\multicolumn{4}{|c||}{ Variations in Product Composition from the Kölbel Reactor (from ref. 19) } \\
\hline Molecular Weight Goal & "Low" & "Medium" & "High" \\
\hline Single-pass $\mathrm{C}_{3}{ }^{+}$product yield $\left(\mathrm{g} / \mathrm{m}^{3} \text { feed) }\right)^{\mathrm{a}}$ & 166 & 175 & 182 \\
\hline Distribution of $\mathrm{C}_{3}{ }^{+}$products $(\%)$ & & & \\
$\mathrm{C}_{3-4}$ & 18 & 7 & 2 \\
Gasoline $\left(\mathrm{C}_{5}-190^{\circ} \mathrm{C}\right)$ & 68 & 40 & 7 \\
Diesel fuel $\left(190-310^{\circ} \mathrm{C}\right)$ & 11 & 26 & 8 \\
$310-450^{\circ} \mathrm{C}$ & 2.5 & 18 & 33 \\
$>450^{\circ} \mathrm{C}$ & 0.5 & 9 & 50 \\
\hline
\end{tabular}

As noted, the production of hydrocarbons per $\mathrm{m}^{3}$ gas increases as the molecular weight of the products increases; however, in no instance does it approach the theoretical yield of $208 \mathrm{~g} / \mathrm{m}^{3}$. On the other hand, Mobil runs consistently produced greater than $200 \mathrm{~g} / \mathrm{m}^{3}$. This low hydrocarbon productivity in Kölbel's work is apparently a problem that many investigators have struggled with.

The quality of a diesel fuel can vary considerably. Cetane number is used as one measure of the quality of a diesel fuel much in the same manner as octane number is used for gasoline. However, octane number and cetane may be viewed as opposites. Thus, highly branched paraffins, olefins and aromatics are desirable, and normal paraffins undesirable, components of a fuel if one wants a high octane number; on the other hand, $n$-paraffins are desirable and highly branched paraffins, olefins and 
aromatics are undesirable components for diesel fuel with a high cetane number. In viewing the high molecular weight product slate in the above table, it is noted that $33 \%$ of the product is diesel and $50 \%$ is heavier molecular weight material that must ultimately be cracked to produce gasoline and diesel. As produced with an iron catalyst, the diesel fraction of the products (straight-run diesel) contains a significant amount of olefins, and consequently a relatively low cetane number. However, when this fraction is hydrogenated it will contain predominantly ( $90 \%$ or greater) $n$-paraffins, and this fraction will have a high cetane number (at or approaching 70). Because there is little difference in the ratio of $\mathrm{i}-\mathrm{n}$-paraffin fraction of the hydrogenated straight-run diesel from an iron catalyst and the straight-run diesel from a cobalt catalyst, the straight run diesel produced by either catalyst will be the same, or very similar. Furthermore, it requires the same amount of hydrogen to produce a paraffin irrespective of whether it is produced indirectly by hydrogenating an initially formed olefin produced by iron catalysis or produced directly using a cobalt catalyst. Based upon straight-run diesel, there should therefore be no difference in the quality of the materials produced using either catalyst. Likewise, the $>450^{\circ} \mathrm{C}$ fraction of the iron and cobalt catalyst is composed essentially of $n$-paraffins, either before or following a hydrogenation step, so that, while the quality of the diesel fuel produced by hydrocracking may depend upon the hydrocracking process utilized, it should not depend upon whether the $>450^{\circ} \mathrm{C}$ fraction is obtained by iron or cobalt catalysis.

It cannot be overemphasized that diesel is not a sufficient specification to use to compare catalysts and/or processes. Straight-run diesel and diesel obtained from hydrocracking will not, in general, have the same properties even when both are composed only of paraffins. The major reason for this is that hydrocracking normally 
produces a significant fraction of monobranched paraffins; in fact, the classical bifunctional hydrocracking mechanism would produce an i-/n-paraffin ratio of 1 or even greater. Thus, it is important, when discussing cetane number, to specify whether one refers to what is straight-run diesel, diesel produced by hydrocracking or some blend of these two products. For blending with petroleum-derived diesel to produce a more environmentally friendly transportation fuel, it is desirable that the F.-T. product have the highest possible cetane number. From the point of view of obtaining a superior diesel for blending with petroleum-derived diesel, it appears that straight-run F.-T. diesel would be preferred over hydrocracked diesel.

In considering the medium and high molecular weight cases shown above, an equal amount of blended diesel would be produced by combining the straight-run and hydrocracked diesel fraction only if the hydrocracking selectivity was such that it produced only $32 \%$ diesel fraction. The selectivity for hydrocracking is much greater than $32 \%$ so that it is obvious that more diesel will be produced from the "high" operation in the above table. However, for a run at La Porte to generate diesel to make a large-scale test, both straight-run and hydrocracked diesel could be produced using either the medium or high molecular weight mode of operation. For the medium molecular weight case the straight-run fraction would dominate over the hydrocracked diesel whereas the opposite would result from the high molecular weight mode of operation.

\subsubsection{Deviations from Anderson-Schulz-Flory}

\subsubsection{Positive Deviations}

Since its introduction about 1950, the Anderson-Schulz-Flory distribution for the products from Fischer-Tropsch synthesis has been accepted. However, only a few 
investigators have been able to attain a product distribution that adheres to this single alpha distribution. Anderson, in his review in the 1950 showed data for products from large scale German and U.S. plants and these exhibited the "double-alpha" plot that has now been reported by many. Donnelly et al. (20) published an approach to calculate the two alphas from the experimental product distribution. Sasol workers report that it is difficult to obtain an accurate evaluation of the higher alpha value because of the small amount of wax production in laboratory studies. These workers report, however, that the Donnelly et al. approach is "suitable for the extrapolation of selectivities," (14). On the other hand, Shell workers (21) report that "In a few hundred independent F.-T. synthesis experiments with various catalyst formulations [iron, ruthenium and cobalt] under different operating conditions it was confirmed that the carbon number distribution were in close agreement with the AFS chain growth kinetics discussed above, with $\alpha$ values varying between 0.7 and 0.95 (Figure 17)." However, these authors did not provide data in their paper (Figure 17) that would cover adequately the carbon number ranges of both alpha values.

${ }^{14} \mathrm{C}$ tracer studies carried out at the CAER using an iron catalyst produced data that led to the postulation of a double alpha ASF distribution with the additional provision that the lower alpha produced all F.-T. products but that the higher alpha pathway produced only alkanes. More recent ${ }^{14} \mathrm{C}$ tracer studies included a measurement in the activity in the higher carbon number alkenes. This more recent data indicate that the ${ }^{14} \mathrm{C}$ distribution in the alkenes is consistent with a single ASF pathway. Accumulation of paraffins in the reactor provide a "product accumulation disguise" so that the second alpha products are due to reactor operation and not to two 
reaction pathways. Thus, based upon CAER data, the observation of a double alpha value is introduced by the operation of the reactor and not by the F.-T. mechanism.

As noted in another section, Sasol workers consider that an iron catalyst can be modified by the use of chemical promoters (e.g., potassium) but that cobalt cannot. On the other hand, the selectivity of cobalt is sensitive to the pressure but not the iron catalyst. Thus, the Sasol workers attribute the observations of the impact of chemical promoters as being due to pressure effects. Thus, they report the chain growth probability as a function of pressure (Figure 18). This translates into the product selectivities shown in Figure 19. It is obvious that if wax is the desired product pressure makes very little difference with the iron catalyst (25 atm appears optimum) but for the cobalt the wax production is still increasing relative to other products even at 40 atm pressure. The Sasol workers report that the wax boiling above $350^{\circ} \mathrm{C}$ can be easily hydrocracked to extinction, yielding about $80 \%$ diesel with a cetane number of at least 70 . 


\subsubsection{Positive Deviation at Higher Carbon Numbers}

There are many examples of this type of deviation and these examples have been produced in many laboratories as well as at large pilot or commercial plants (22). A number of reasons have been advanced to account for this distribution. Included among these are two or more chain growth pathways, the impact of alkali, and alkene reincorporation.

A more logical explanation for the deviation is reactor operation and the hold-up of heavier materials.

\subsubsection{Cut Off of Product Distribution}

At the start-up of a slurry or even fixed-bed reactor a period of time is required until the vapor-liquid equilibrium is established. The length of time that it takes to reach vapor-liquid equilibrium depends upon carbon number; the higher the carbon number the longer the time it takes. Satterfield, Bell and others have shown the impact of this factor. This effect is a result of normal reactor operation and is independent of F.-T. selectivity deviations.

\subsubsection{1. $\underline{\text { Chinese }}$}

Yang et al. (23) considered literature reports and proposed a new product distribution formulation. They proposed that ASF growth was followed but that on any metal crystal size, only molecules with a lower carbon number than the cut-off value could be produced. They fit an equation they derived to three sets of data and showed a good correlation between theory and experimental data. One set of data was for a series of carbon supported iron catalysts that had different distribution of metal crystallite sizes (24). There are two problems with the use of these data: (1) the metal crystallite size will have little meaning since under reaction conditions the supported Fe 
crystallites will be converted to iron carbide and/or iron oxide and (2) products were analyzed by an on-line g.c. with a transfer line that was heated only to a temperature that is consistent with the cut-off of products being due to vapor pressure effects rather than metal crystallite size. A consideration of other data (25-35) show that the selectivities were most likely due to operational rather than F.-T. mechanistic factors.

Thus, while many accounts have been provided to show that cut-off has been accomplished, none of these studies have been conducted under realistic conditions for a sufficient length of time to ensure that liquid-vapor equilibrium had been established.

\subsubsection{Syntroleum}

The Syntroleum Process involves the conversion of gas to liquids and offers a variety of options. Agee (36) reports that work began on a program to produce a catalyst that limits the growth of hydrocarbon chains to eliminate wax production and at the same time minimizing the production of light hydrocarbons $\left(\mathrm{C}_{1}-\mathrm{C}_{4}\right)$. Agee reports that multi-week test runs in a fluid bed reactor at the pilot plant yielded a product profile that indicates success. Based upon data presented at the AIChE meeting, and repeated at the Spring ACS meeting (37), the $\mathrm{C}_{1}-\mathrm{C}_{4}$ gases are low, and the products cut off by carbon number 25 . The most surprising feature of this example is that there is a linear decline in the products with increasing carbon number in the $C_{1}-C_{4}$ range. This cannot happen in a normal polymerization reaction unless the higher carbon number components also continue a similar decline. Agee indicates that the new chain-limiting catalyst eliminates the need for a hydrocracking step; presumably he did not eliminate hydrocracking as an operation that is combined with F.-T. However, this would have to be a new kind of hydrocracking since significantly more $\mathrm{C}_{4}$ is produced 
than $\mathrm{C}_{1}-\mathrm{C}_{3}$ during normal hydrocracking. Based upon currently practiced petroleum technology, it appears that the only way that the distribution shown Figure 20 is possible, would be for the $\mathrm{C}_{2}-\mathrm{C}_{4}$ products to be converted to higher carbon number materials. This would imply that either the $\mathrm{C}_{2}-\mathrm{C}_{4}$ fraction is primarily alkenes and that these alkenes are in some manner caused to reincorporate in the F.-T. process to produce higher carbon-number products or to be oligomerized by some proprietary catalyst; this would explain the absence of the usual amounts of the $\mathrm{C}_{2}-\mathrm{C}_{4}$ fraction but still would not explain how these authors are able to terminate chain growth. If on the other hand, the products from the F.-T. step are not olefinic, then olefin reincorporation cannot explain their results and they would have to have discovered some new catalysis that will activate saturated alkane hydrocarbons, something that is being widely investigated today but so far with little success. In any event, the Syntroleum process produces a better product distribution than the very severe hydrocracking of F.-T. wax in the Shell process (Figure 21). At this time, Syntroleum has non-exclusive licensing agreements with three companies: Marathon Oil Co., Texaco, Inc. and Arco.

\subsection{Slurry-Wax/Catalyst Separation}

\subsubsection{British}

The catalyst, after "break-in," was 1-3 $\mu \mathrm{m}$ in size. A liquid slip-stream was withdrawn continuously from the reactor and catalyst was recovered in a multiple-stage, gravity-settling apparatus. Because of the relatively rapid catalyst aging rates as well as significantly coke formation, reliable data for wax/catalyst separation is not available.

\subsubsection{Kölbel}


A finely divided powder catalyst was utilized; the starting material has a particle size $<30 \mu \mathrm{m}$ but sizes during or after use is not provided. The liquid level in the reactor was maintained by a float-device. A slip-stream, utilized when excess liquid was produced, allowed for wax/catalyst separation by pressure filtration. [Kölbel operated most of the times under conditions where the liquid inventory of the reactor could be maintained only by adding heavier liquid products along with the synthesis gas and wax/catalyst separation was not a problem.] As an alternative, wax/catalyst separation could be effected by centrifugation. Capability for replacement of the catalyst was included in the process although catalyst replacement rates are not given. Data are not available to enable one to reach valid conclusions about the effectiveness of the wax/catalyst separation because of the lack of knowledge of catalyst addition rates. Based on the data in Table (Kölbel) for the "low" operating conditions, an upper limit of $15 \%$ reactor-wax removal can be set, and in practice it should have been much lower.

\subsubsection{U.S. Bureau of Mines}

The U.S. Bureau of Mines operated a $7.6 \mathrm{~cm} \mathrm{ID} \mathrm{x} 3.05 \mathrm{~m}$ high slurry reactor (e.g., 38). This unit utilized a parallel downflow slurry recycle line that was equipped with a porous metal filter. During a 52 day operating period, upsets in maintaining slurry circulation through the recycle line were encountered. It is not known whether this effect was responsible for the catalyst activity decline that occurred during the period of operation. For either the precipitated or fused iron catalyst, the original oxidic material was ground to provide $<60 \mu \mathrm{m}$ particles; following use the particle size was reduced to about $1 \mu \mathrm{m}$.

\subsubsection{Mobil Oil}


Mobil operated a slurry reactor and performed 13 runs during the course of its

DOE contract (18). A simplified flow diagram of the two-stage plant is shown in Figure

13. During this run it is reported that:

"The improved on-line F.-T. reactor-wax separation system enabled us to increase the flexibility and reduce the manpower requirement for the reactor-wax/slurry separation. A schematic of this system is shown in Figure 14. During normal operation, slurry is withdrawn continuously from the F.-T. reactor at the $610 \mathrm{~cm}$ level [762 cm to start of the conical shaped reactor area in Figure 14], and entrained gas is disengaged in a small disengager pot which is connected to the reactor-top. The gas-free slurry is passed through a dip-tube into a two-liter settling pot. The dip-tube length is designed to maintain $80 \%$ of the settling pot volume above its tip. The concentrated slurry exits the settling pot through a conical section, and is pumped back to the slurry reactor at the $305 \mathrm{~cm}$ level through a positive-displacement slurry pump. The pump is inverted, i.e., feed enters at the top and effluent exits at the bottom. This prevents catalyst settling in the feed line to the pump, but requires spring loading of the pump check valves. The clean reactor-wax is withdrawn from the top of the settling pot either semi-continuously by periodically opening a valve, or it can be withdrawn continuously by using a metering valve."

It was reported that the clean reactor-wax contained $0.03 \%$ solids. During this operation the reactor slurry was reported to contain $25-27 \mathrm{wt} \%$ solids. This means that 
$0.12 \%$ of the solids in the reactor are removed in the clean wax. While this type of reactor-wax/catalyst separation may work at the pilot plant, it appears that the separation/reactor volumes would be very large for a commercial plant reactor.

\subsection{5. $\underline{\text { Sasol }}$}

In a patent specification, Sasol workers define a wax/catalyst separation device and operational procedures for its use (Figure 22). A number of these separation devices are located in the reactor slurry. Reactor-wax can be withdrawn through the separation device; depending upon the catalyst size distribution some portion of catalyst fines will be removed together with the reactor-wax. The design of the separation device is illustrated in Figure 23. A unique feature of this design is the use of a trapezoidal shaped wire utilized so that the filter exposes the smaller opening-size to the catalyst/wax slurry side and its larger opening-size to the clean reactor-wax side. This design is utilized so that any blockage of the separation device opening occurs on the catalyst/reactor wax side and can be easily removed by over-pressuring on the clean wax side at appropriate intervals. A unique feature of this design is that any particle that passes through the opening on the catalyst/reactor-wax side will be able to traverse the pore without being impeded by a pore constriction.

\subsubsection{Exxon}

Included in the more than 200 patents issued to Exxon is one that describes "a reactor housing having a plurality of reaction tubes vertically disposed therein for conducting slurry phase hydrocarbon synthesis reactions under substantially plug flow conditions, and wherein provision is made for uniformly distributing gas bubbles in slurry liquid into the reaction tubes (Figure 24). 
Included in this patent is a description of a reactor-wax/catalyst separation device. This device is described thusly (39):

"...Above the liquid space is another tube sheet $\mathbf{3 0}$ holding filter cartridges $\mathbf{3 1}$ which may contain sintered metal mesh, woven metal fibers, glass fibers, cloth, fibrous carbon that can remove the catalyst particles while allowing passage of the liquid. The filter cartridges are each vertically aligned with each reaction zone and prevent catalyst particles from reaching the upper portion of the housing 8. Above the filter cartridge tube sheet is a gas-liquid disengagement zone $\mathbf{3 6}$ topped by a foamy interface $\mathbf{3 8}$. Liquid product from the hydrocarbon synthesis may be removed via line $\mathbf{4 0}$, or alternately vis line $\mathbf{3 3}$ at or above the filter cartridge tube sheet. A demister $\mathbf{4 1}$ finally separates gas from liquid droplets and residue gases are withdrawn via line $\mathbf{4 2}$. Thus, the liquid space above the upper tube ends and below the filter tube sheet allows fluid communication between the upper tube ends and the alternate slurry addition/removal conduit $\mathbf{3 2}$ as well as the space above the filter tube sheet. The space above the filters and filter tube sheet allows fluid communication of the gas outlet means, the liquid outlet means and the space below the filter tube sheet, thereby further allowing fluid communication to the upper ends of the reaction tubes." 


\subsubsection{Statoil}

Patent applications by Statoil describe a recent report identified only as "a report issued by the United States Department of Energy." The DOE report addressed the question of catalyst/wax separation in Fischer-Tropsch slurry reactor systems and concludes:

"Internal filters immersed in the reactor slurry, as used in some bench-scale or pilot-scale units, do not work successfully due to operational difficulties. A reactor with a section of its wall as a filter may be operable for a pilot plant but is not practicable for commercial reactors. Internal filters are subject to plugging risks, which may cause premature termination of the run, and commercial plants are not allowed to take chances."

The patent applicants have discovered that, contrary to the teachings of the DOE report, "...it is possible to provide a continuous reaction system for a FischerTropsch synthesis in which it is not necessary to perform the solid/liquid separation in an external filter unit. Furthermore, a sufficiently high flow rate of filtrate for commercial operation can be achieved." (40). The drawings included in these applications are similar to the word description and/or the schematic drawings in the above patents.

The only examples of actual operation given in the Statoil patent applications are for a cold model using oil, alumina and nitrogen gas. The cold model was operated for about 40 hours without major plugging problems that inhibit liquid flow. However, it does not appear that the applications provide any data to substantiate the claims that they have overcome the problems enumerated in the DOE report.

3.7.8. China 
The operators encountered problems in separating reactor-wax from the catalyst slurry in both the bench scale and the large plant. The data reported for the small bench scale plant indicated that the reactor wax discharged contained consistently 7.5 wt. \% catalyst. Considering that the reactor contained a slurry with $12 \mathrm{wt} . \%$ catalyst, the separation was not very efficient. These workers indicate that the catalyst loss is the reason for the decline in activity and state that "...if we solve the problem of catalyst loss, long-term operation is very possible." (7).

\subsection{Process Considerations}

The relative usage of hydrogen and carbon monoxide depends upon the $\mathrm{CO}$ conversion level (Figure 10). A similar curve has been obtained by UOP workers (41). Thus, the hydrocarbon productivity per gram of iron and per reactor volume is higher at lower $\mathrm{CO}$ conversion levels. Furthermore, the fraction of $\mathrm{CO}$ that is converted to hydrocarbons is greater at lower CO conversion levels. Thus, up to CO conversion levels of about $50 \%$, the iron and cobalt catalyst will exhibit similar activity and hydrocarbon distribution selectivity properties. However, the iron catalyst operated at $50 \%$ or lower CO conversion will produce a very olefinic product. From the point of view of chemicals, such as could be utilized with a pioneer plant, the iron catalyst would provide a significant advantage. In fact, iron would be the preferred catalyst for any operation where chemicals would be a serious consideration.

Thus, in order to take full advantage of the activity and selectivity properties of the iron catalyst, it should be utilized in a process employing recycle or multiple reactors. It would appear that a process utilizing multiple reactors with water knock-out between reactors and the addition of make-up syngas, would be the preferred option. 
An additional advantage of the iron catalyst is that it can be operated at a higher temperature than the cobalt catalyst. This would permit the generation of higher quality steam and with the iron catalyst the generation of electricity could be considered as an approach to utilize some of the energy rejected during the hydrocarbon synthesis step.

\subsection{Supercritical Phase Fischer-Tropsch Synthesis}

It is claimed that three main advantages of the supercritical synthesis are: (1) rapid diffusion of reactants, (2) effective removal of reaction heat and (3) effective extraction of wax, $\alpha$-olefins and water. Thus, the supercritical operation could, if item 3 is accomplished, decrease or eliminate secondary reactions.

Yokota et al. (42) compared the results of the operation of three types of reactors: fixed-bed, liquid and supercritical. In order to make an effective comparison the feed consisted of 22-36\% synthesis gas with the remainder being diluent (nitrogen for the fixed-bed, hexadecane and nitrogen for the liquid, and n-hexane for the supercritical). An iron catalyst was utilized and had the following composition by wt.

fraction: $\mathrm{Fe}, 83.5 ; \mathrm{Ca}, 2.1 ; \mathrm{Al}, 1.5 ; \mathrm{Si}, 0.4 ; \mathrm{K}, 0.5$ or $\mathrm{Fe}, 99 ; \mathrm{Cu}, 0.3 ; \mathrm{K}, 0.3$. Thus, both iron catalysts were of the low alpha type utilized by Mobil Oil and La Porte Run II. The total pressure was $5 \mathrm{MPa}$ (about 50 atm.; $728 \mathrm{psi}), 270^{\circ} \mathrm{C}, \mathrm{H}_{2} / \mathrm{CO}=1$ and $\mathrm{W} / \mathrm{F}(\mathrm{CO}+$ $\left.\mathrm{H}^{2}\right)=10 \mathrm{~g}$-cat $\mathrm{h} / \mathrm{mol}$. Each run was conducted for 6 hours. Under no circumstances should it be considered that a steady-state operation was attained.

The authors show the $\mathrm{CO}$ conversion, $\mathrm{CO}_{2}$ yield and chain-growth probabilities for the fixed-bed, supercritical and slurry phase reactors as 33.0, 30.2 and $27.9 ; 8.65$, 7.52 and 9.15 ; and $0.84,0.83$ and 0.80 , respectively. The authors considered these differences to be significant and attribute the lower $\mathrm{CO}_{2}$ yield for the supercritical 
operation as being due to the increased removal of water from the reactor. The reported chain growth probabilities appear high for an iron catalyst that contains such a low level of potassium. These data should be viewed as suggestive at best.

Fujimoto et al. (43) report that the addition of a small amount of heavy 1 -olefin in a supercritical-phase or liquid-phase F.-T. reaction medium greatly enhanced the selectivity of wax products, with increased $\mathrm{CO}$ conversion and suppressed methane selectivity. A cobalt-silica catalyst that contained La was used. The authors reported that the addition of 1-tetradecene or 1-hexadecene significantly decreased the hydrocarbon production for carbon-number products lower than that of the added alkene, and increased significantly the production rate of the carbon-number products with higher carbon numbers that the added alkene (Figure 25). The impact shown in Figure 25 is astounding. When alkenes were not added the production rate of the products above carbon number 15 decreased with increasing carbon number; this is expected and observed in normal F.-T. synthesis. However, when the alkene is added, the hydrocarbon production with carbon-numbers above that of the alkene become essentially constant; i.e., independent of carbon number. It appears that this requires the added alkene to initiate chain growth that differs from that of the F.-T. reaction. If the only impact of the alkene was to initiate additional growing chains, the product distribution above the carbon number of the added alkene should remain the same; i.e., the rate of production of all carbon-number products should increase but should still be produced in the same ratio as they were when no alkene was added. The data obtained when 1-heptene, in contrast to 1-tetradecene or 1-hexadecene was added, is in better agreement with the expectation. 
Lang et al. (44) utilized a precipitated iron catalyst in a fixed-bed reactor and found that the catalyst activity and lumped hydrocarbon product distribution under the supercritical conditions were similar to those obtained during reaction at the baseline (non-supercritical) conditions. This is in contrast to the views expressed above. They did report slightly higher selectivities for the 1-alkenes during supercritical operation (Figure 26). They indicate that this suggests that the F.-T. reaction is not diffusionally limited under their reaction conditions. The higher alkene production during supercritical operation was due to higher diffusivities and desorption rates of the high molecular weight olefins relative to those under normal F.-T. conditions. 


\section{References}

1. B. Jager, AIChE Mtg., Houston, 1997.

2. B. Jager and R. Espinoza, Catal. Today, 23 (1995) 17.

3. R. Srinivasan, L. Xu, R. L. Spicer, F. L. Tungate and B. H. Davis, Fuel Sci. Tech. Int., 14 (1996) 1337.

4. T. Kuntze, K. Hedden and A. Jess, Erdöl Erdgas Kohle, 111 (1995) 67.

5. "Report on the Petroleum and Synthetic Oil Industry of Germany,: Ministry of Fuel and Power, His Majesty's Stationery Office, London, 1946, pp 96-100.

6. C. B. Benham, M. S. Bohn, and D. L. Yakobson, U.S. Patent 5,324,335, June 28, 1994.

7. $\quad$ "Synthesis of Liquid Fuels From Coal," (Bijiang Zhang, Ed.), Science and Technology Publishing Corp., Shanxi Province, China, 1993.

8. L. Koenig, et al., Bet. Bunsenges. Phys. Chem., 91 (1987) 116.

9. B. Cornils, W. A. Herrmann and M. Rash, Angew. Chem., Int. Ed. Engl., 33 (1994) 2144.

10. B. H. Davis, "Gulf-Badger - Review, April, 1977.

11. B. H. Davis, Statoil Report, June, 1997.

12. E. Iglesia, S. C. Reyes, R. J. Madon and S. L. Soled, Advan. Catal., 39 (1993) 321.

13. B. H. Weil and J. C. Lane, "Synthetic Petroleum from the Synthine Process, Remsen Press, Chem. Pub. Co., Inc., New York, 1948, p 67.

14. P. J. van Berge and R. C. Everson in "Natural Gas Conversion IV," (M. de Pontes, et al., Eds.), Elsevier, Amsterdam, pp. 207-212.

15. H. Kölbel and M. Ralek, Catal. Rev.-Sci. Eng., 21 (1980) 225. 
16. J. M. Fox, III and S. S. Tam, Topics in Catal., 2 (1995) 285.

17. J. R. Inga and B. I. Morsi, 13th Ann. Int. Pittsburgh Coal Conf., 1996, pp. 924929.

18. J. C. W. Kuo, DOE/PC/60019-9, October, 1985.

19. M. L. Poutsma, ORNL-5635, February, 1980.

20. T. J. Donnelly, C. N. Satterfield and I. C. Yates, Energy \& Fuels, 2 (1977) 313.

21. J. Eilers, S. A. Posthuma and S. T. Sie, Catal. Lettr., 7 (1990) 253.

22. B. H. Davis, ACS Div. Fuel Chem., 37 (1992) 172.

23. Y. Yang, S. Pen and B. Zhong, Catal. Lettr., 16 (1992) 351.

24. V. K. Jones, L. R. Neubauer and C. H. Bartholomew, J. Phys. Chem., 90 (1986) 3832.

25. T. Mitsudo, H. Boku, S. Murachi, A. Ishihara and Y. Watanabe, Chem. Lettr., (1985) 1463.

26. R. Snel, Catal. Lettr., 1 (1988) 327.

27. D. Fraenkel and B. C. Gates, J. Am. Chem. Soc., 102 (1980) 2478.

28. D. Fraenkel and B. C. Gates, U,S. 4,294,725; assigned to U. Delaware.

29. D. Balleret-Tkatchenko and I. Tkatchenko, J. Mol. Catal., 13 (1981) 1.

30. L. F. Nazar, G. A. Ozin, F. Hugues, J. Odber and D. Rancourt, J. Mol. Catal., 21 (1983) 313.

31. H. H. Nijs, P. A. Jacobs and J. B. Utterhoeven, J. Chem. Soc., Chem. Commun., (1979) 1095.

32. H. H. Nijs, P. A. Jacobs and J. B. Utterhoeven, J. Chem. Soc., Chem. Commun., (1979) 180.

33. I. R. Leith, J. Chem. Soc., Chem. Commun., (1983), 93. 
34. R. K. Unger and M. C. Baird, J. Chem. Soc., Chem. Commun., (1986), 643.

35. D. Vanhove, P. Makambo and M. Blanchard, J. Chem. Soc., Chem. Commun., (1979), 605.

36. M. A. Agee, AIChE Meeting, Houston, March 1997.

37. M. A. Agee, ACS Meeting, Div. Fuel Chem. Preprints, April 1997.

38. M. D. Schlessinger, J. H. Crowell, M. Leva and H. H. Storch, Eng. Process Dev., 43 (1951) 1474.

39. R. M. Koros, U.S. Patent 5,384,336, January 24, 1995.

40. Int. Pub. No. WO 90/07377, 12 July 1990; Int. Pub. No. WO 96/06683, 7 March, 1996; U.K. Appl. GB2,299,767A, 16 October, 1996; U.K. Patent Appl. GB2,281,224A, 1 March 1995; Int. Pub. No. WO 94/16807, 4 August 1994.

41. V.U.S. Rao, G. J. Stiegel, A. C. Bose, G. J. Cinquegrane and R. D. Srivastava, ACS Div. Fuel Chem., 37 (1992) 184.

42. K. Yokota, Y. Hanakata and K. Fujimoto in "Natural Gas Conversion," (A. Holmen et al., Eds.), Elsevier, Amsterdam, (1991), pp. 289-295.

43. K. Fujimoto, L. Fan and K. Yoshii, Topics in Catal., 2 (1995) 259.

44. X. Lang, A. Akgerman and D. B. Bukur, Ind. Eng. Chem. Res., 34 (1995) 72. 


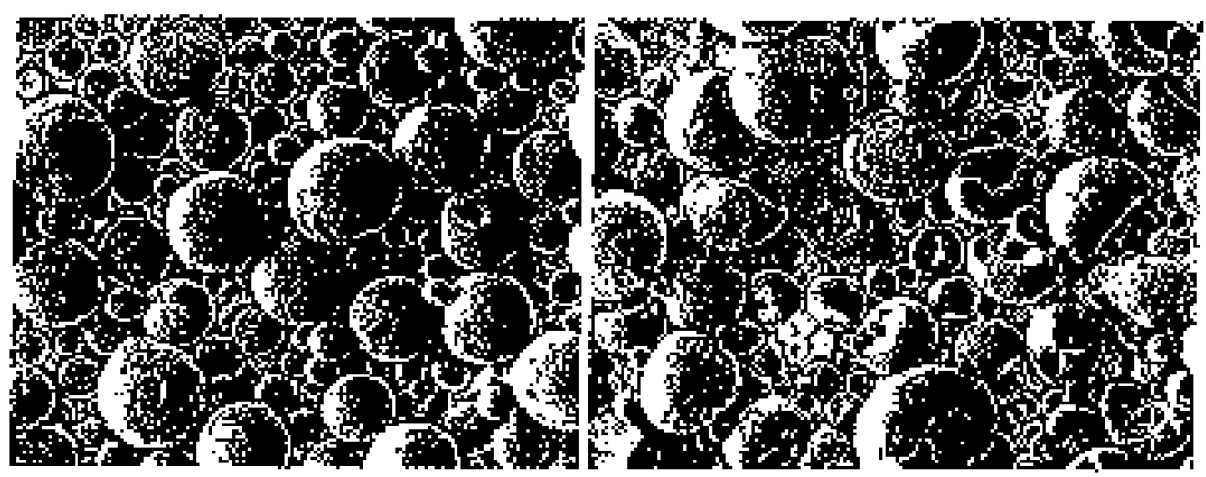

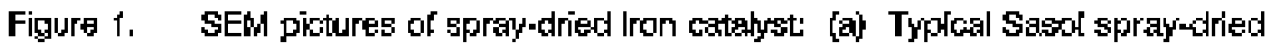

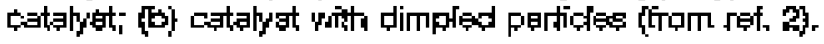
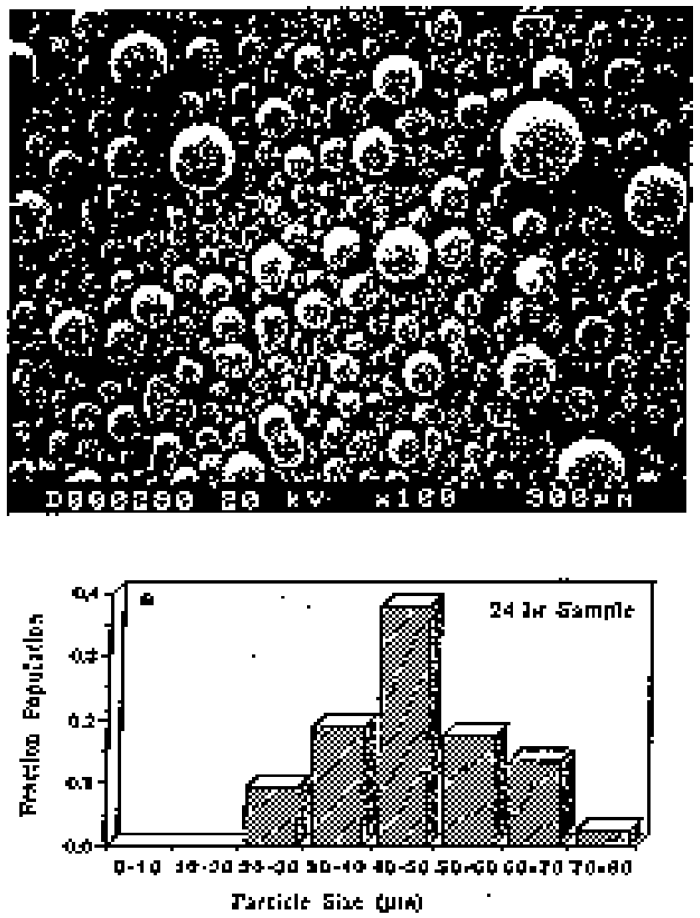

Figure 2. Sosming elactron mictograph from the trol sample after carbiding ard

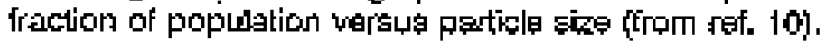




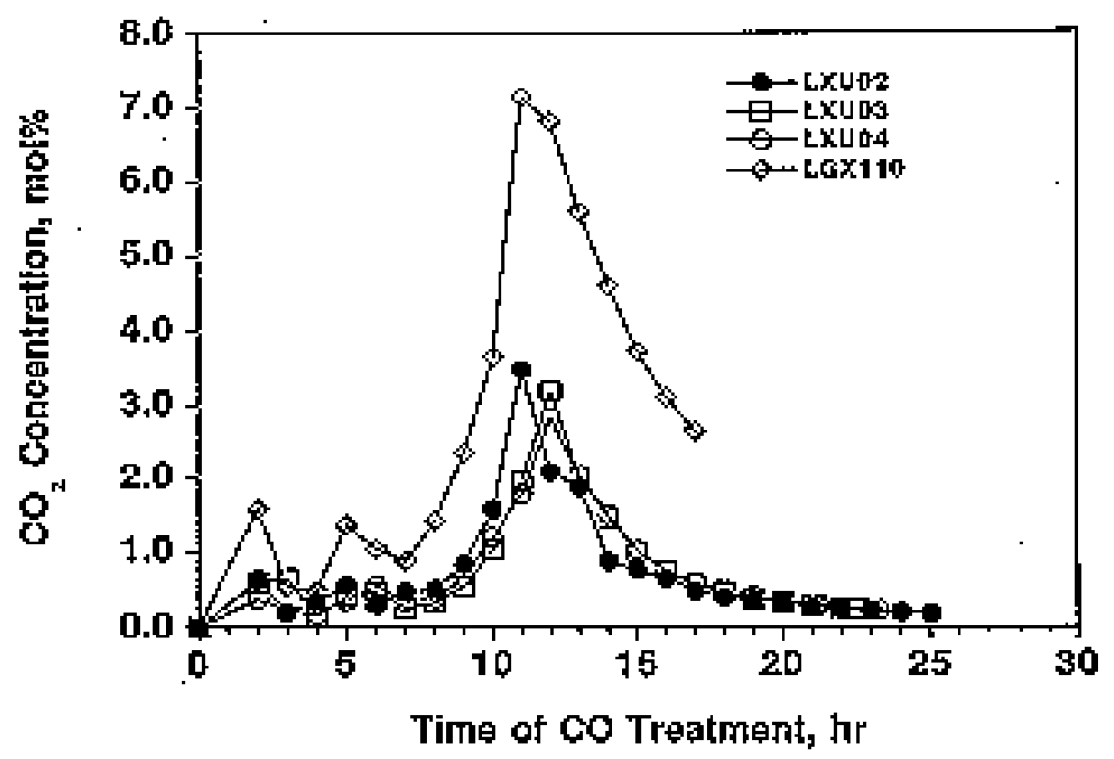

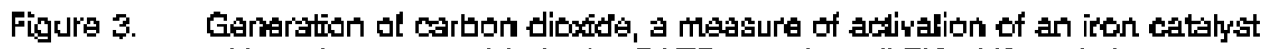
with carbon monoside in the CAEF autoctase [LEC 110\} and slumy butble wolumn (LX'S runs) reactor.

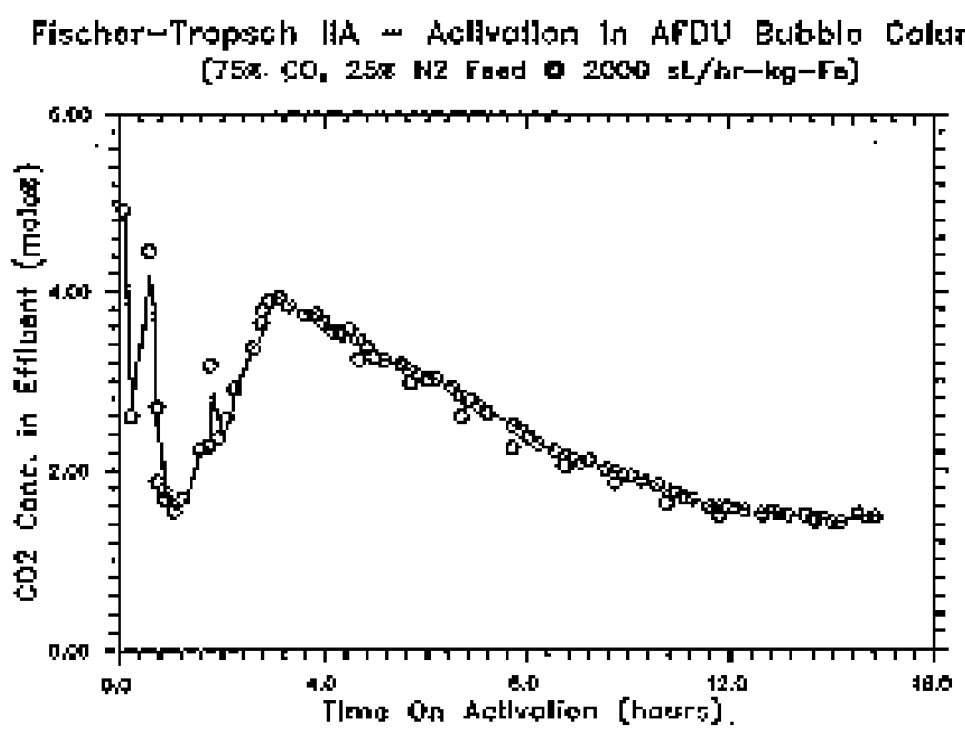

Figure 4. Aotiution of the iron tatelyst prepared by Unitad Cetalysta, Ino. in the La Porte Eurn II. 


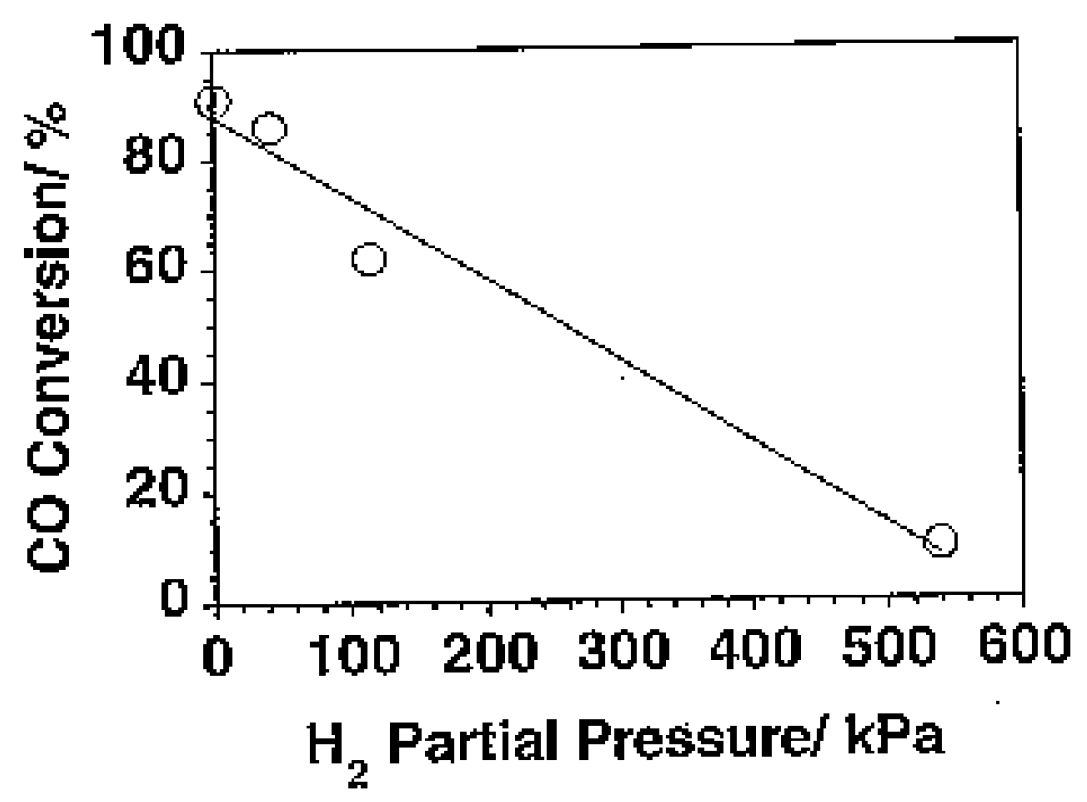

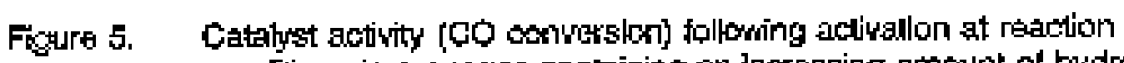

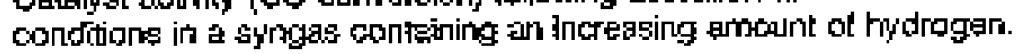

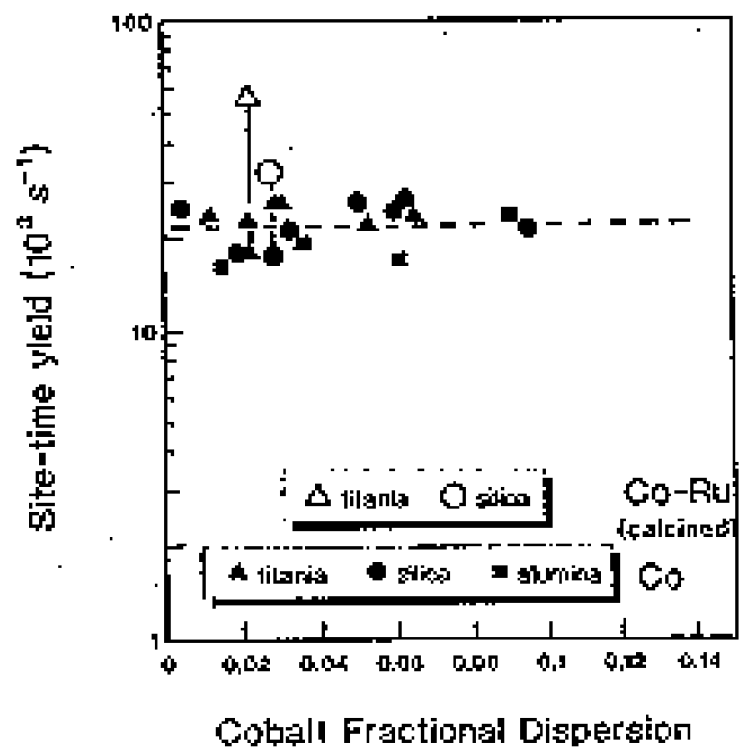

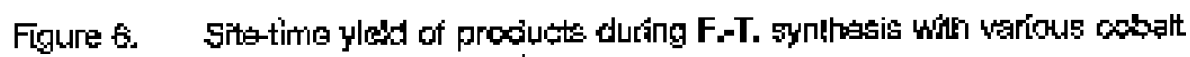
catalysts (firom ㅂef. [?]). 


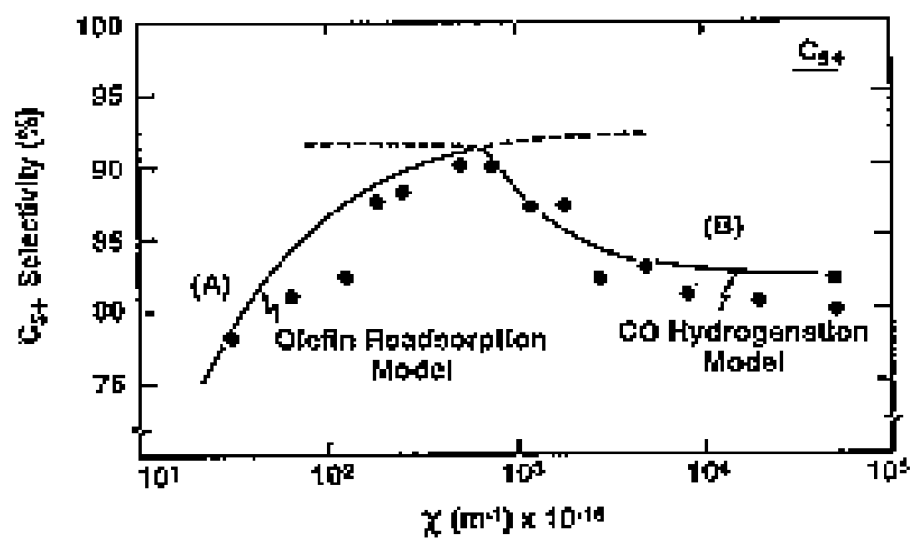

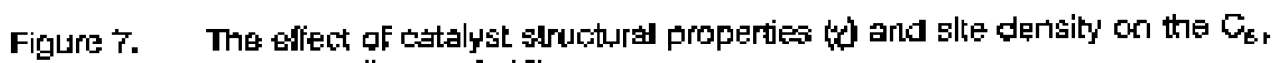
steletiwty [irom ref, i2].

Is WWG

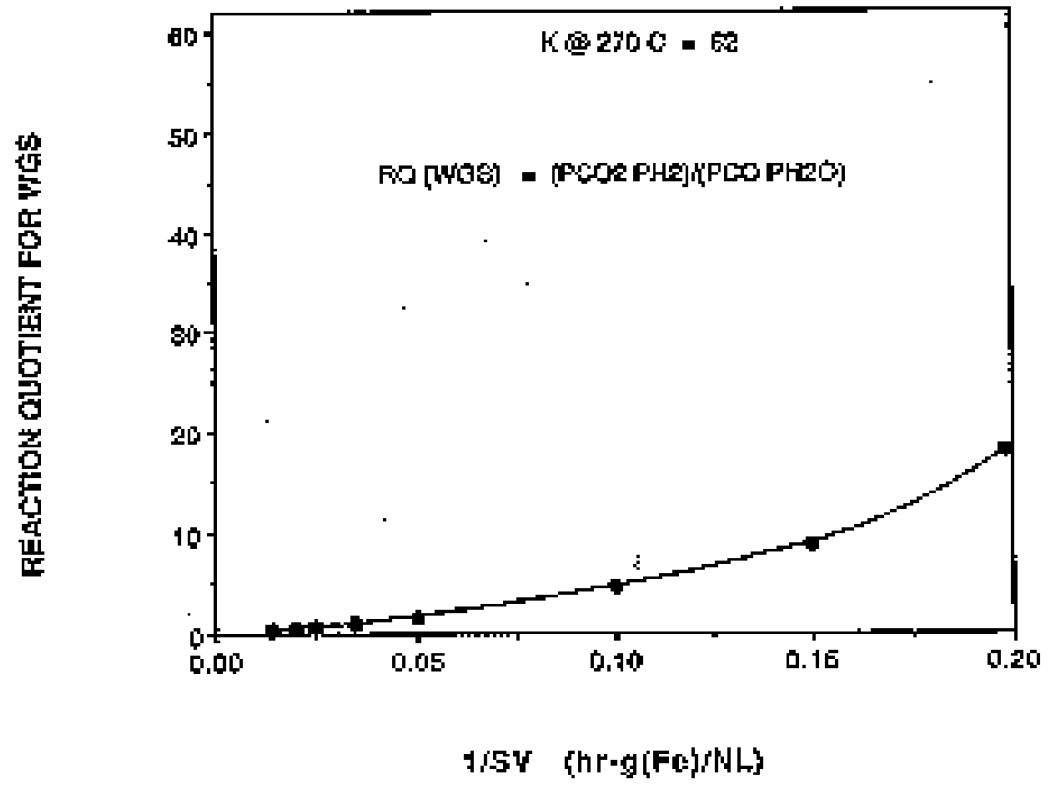

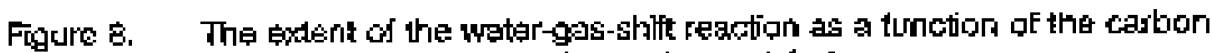

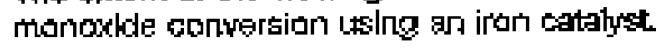




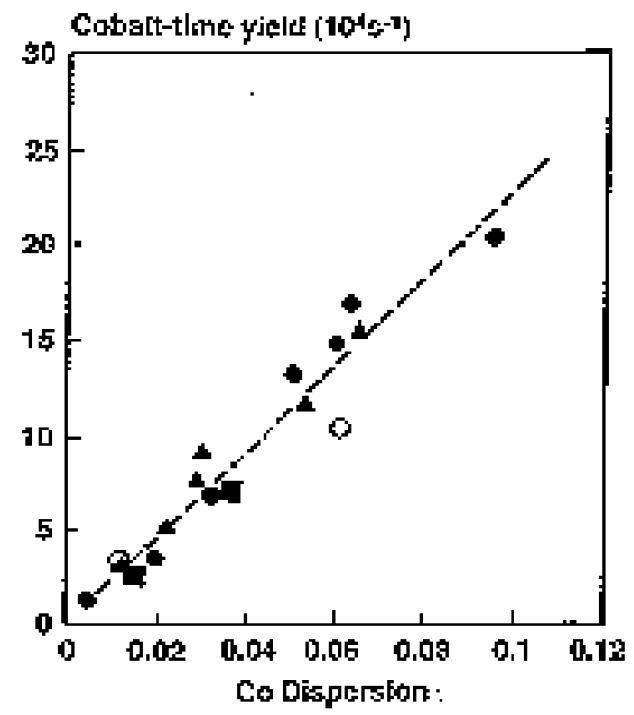

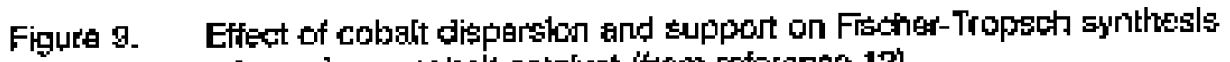
rates Lting a coball catalysit [tom reference 12].

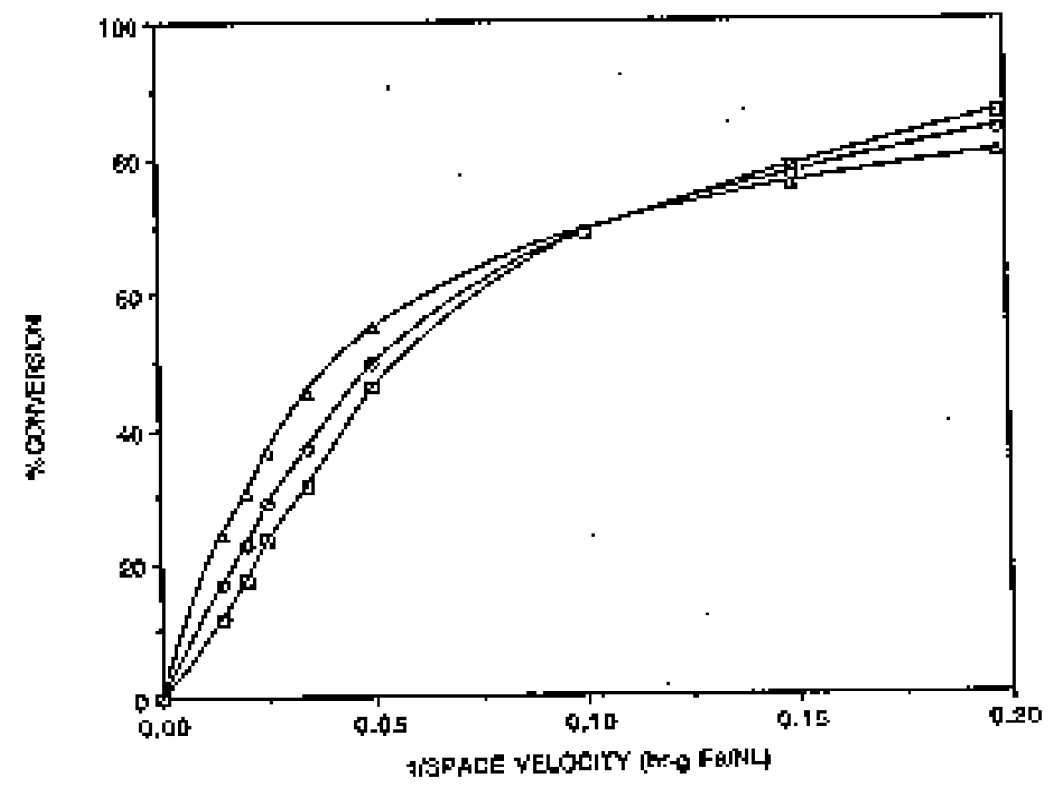

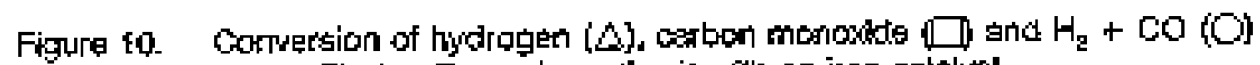
duting Fischer-Tedp'sch syrithesis with an iron colalyst 


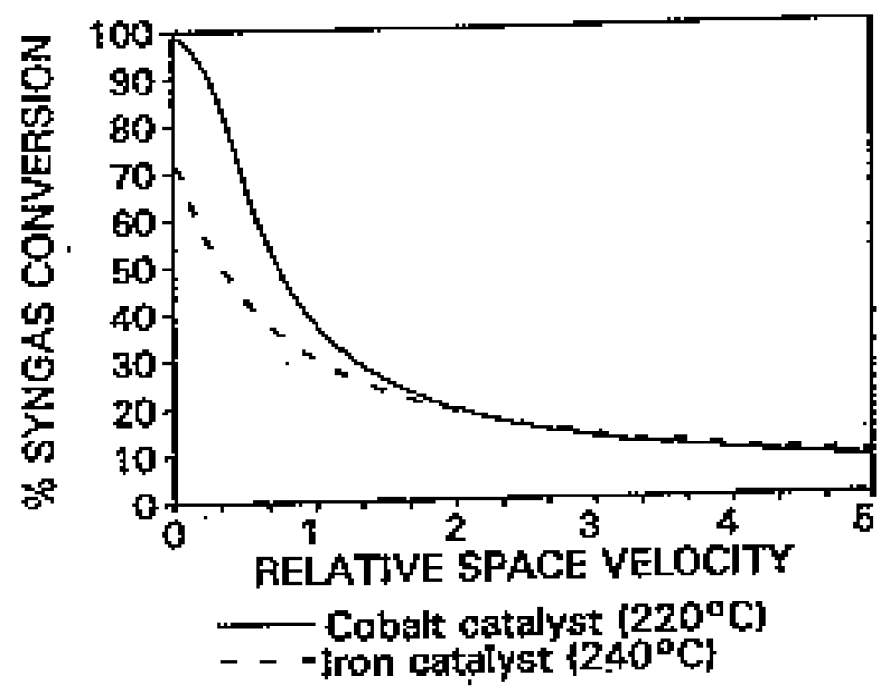

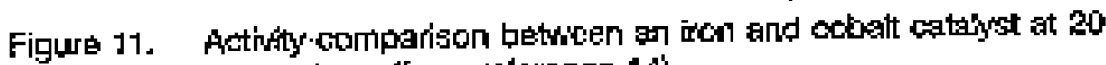
atmosphare (from tolerene 14).

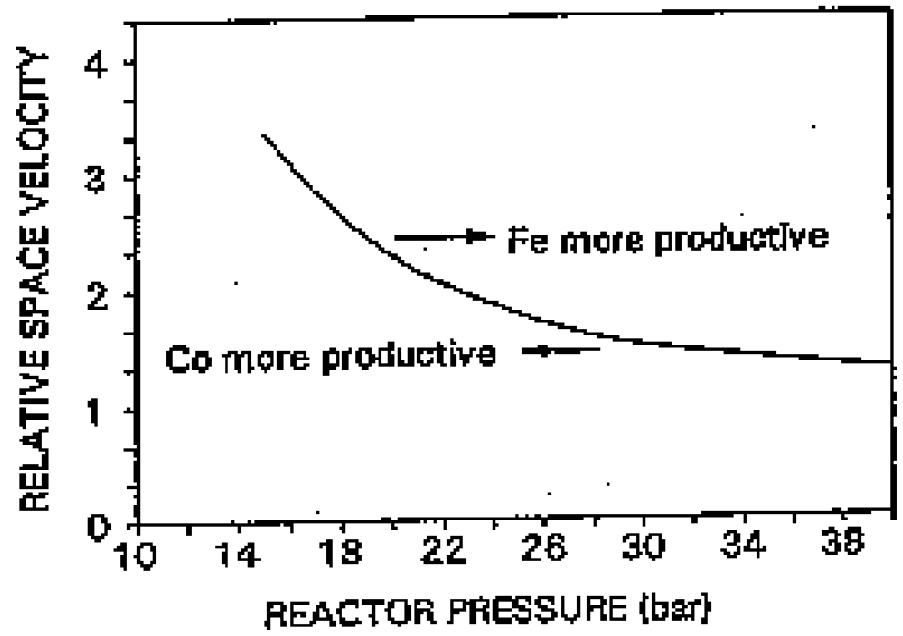

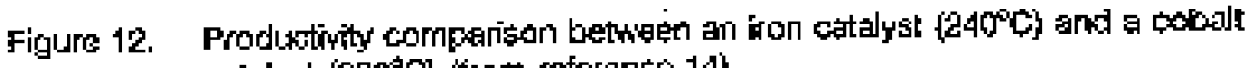

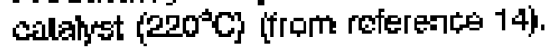




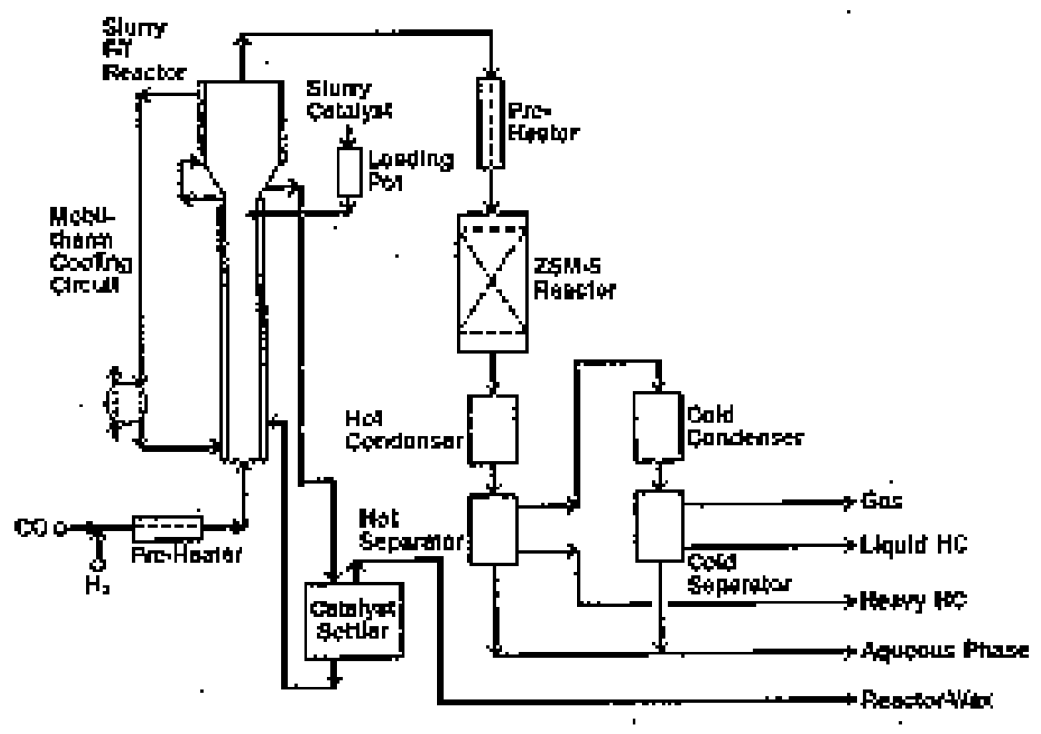

Frgure 13. Simplited fow diagram of the Mobil of hwo-stabs plant for gynthests gas conversion (fiom eefarence 18).

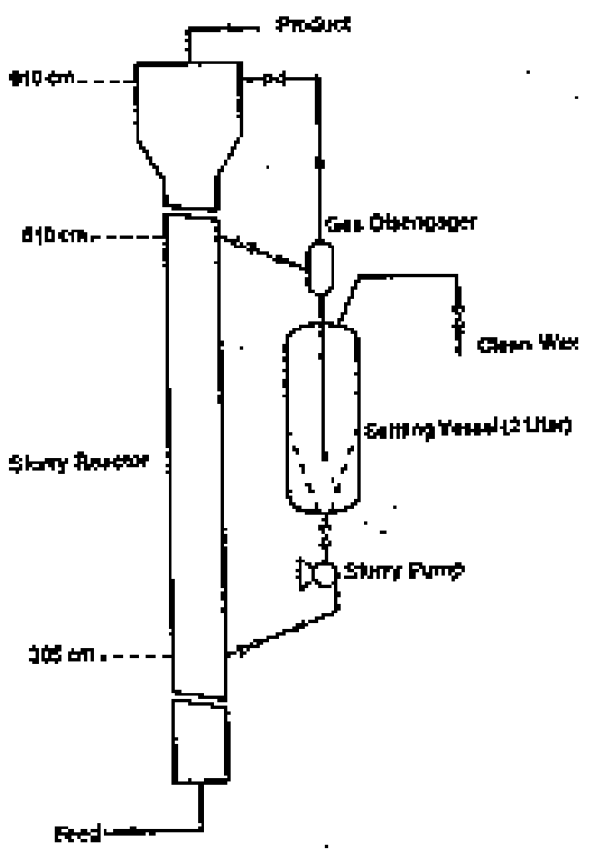

Figare 14. Sohematio diagram of the continuous setting sysam for cotalystiwax separation (crom reference 19). 


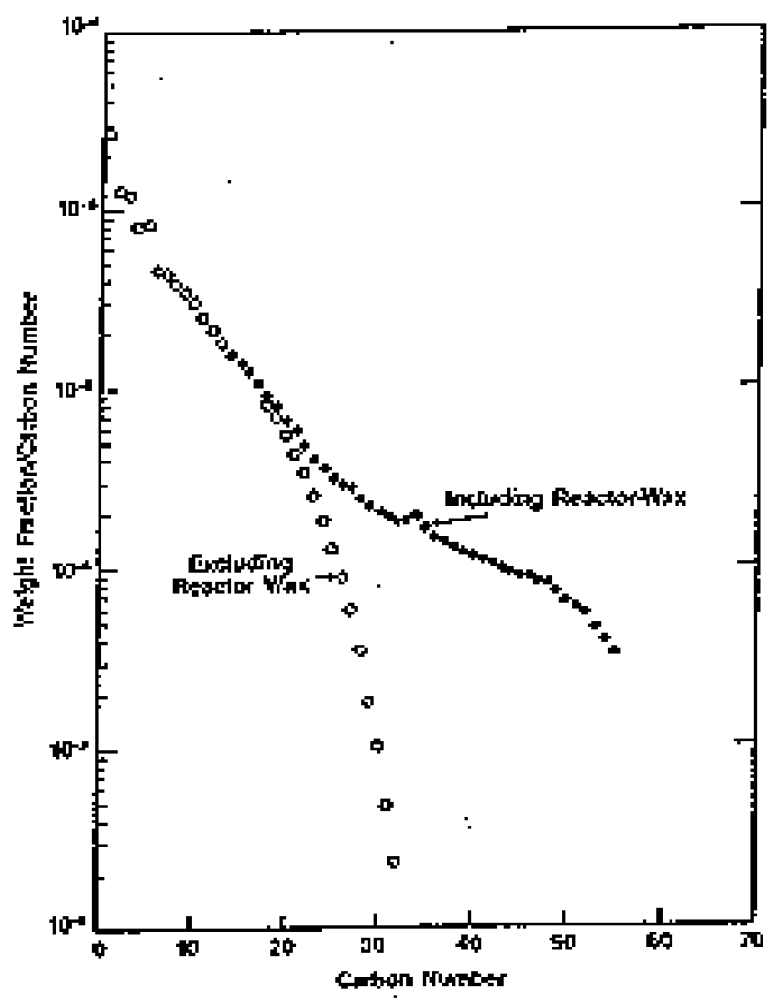

Figure 15. Schulz-Fiory distibution for the first stage Fisctier-Tropsch products obtained by Mabil Oil al a teactor wax yild of 48 wtos (from reference 16).

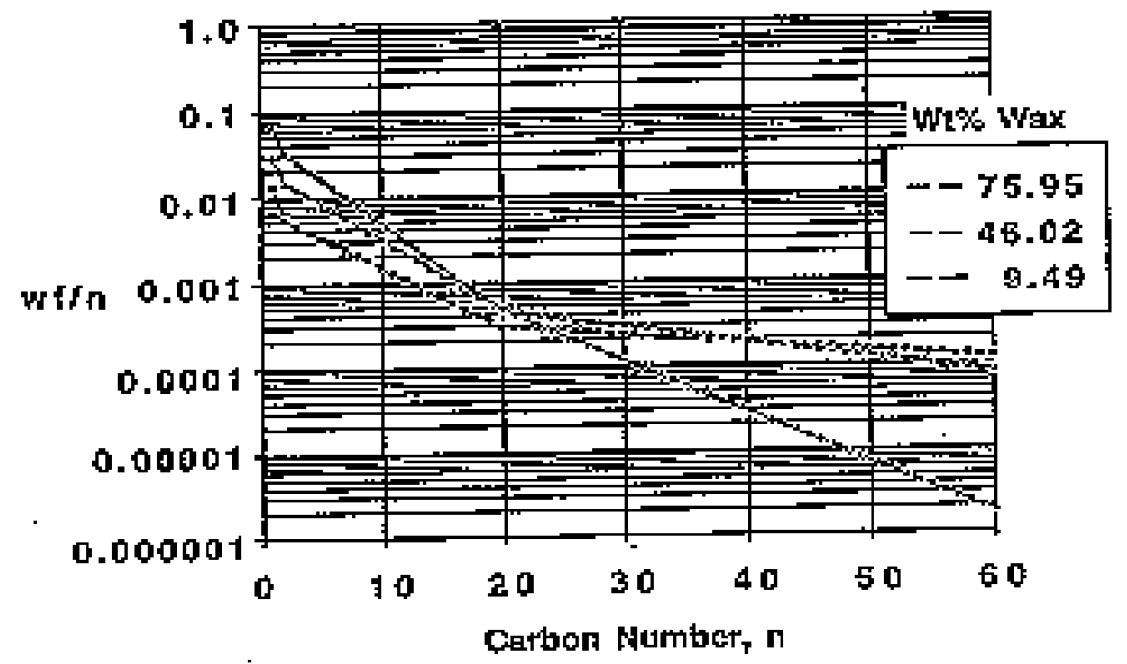

Figure 16. Sohulz-Fory distnbution based on Mobat of dazla al low, intermediato and

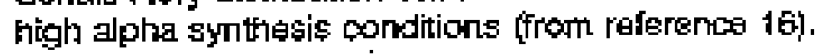




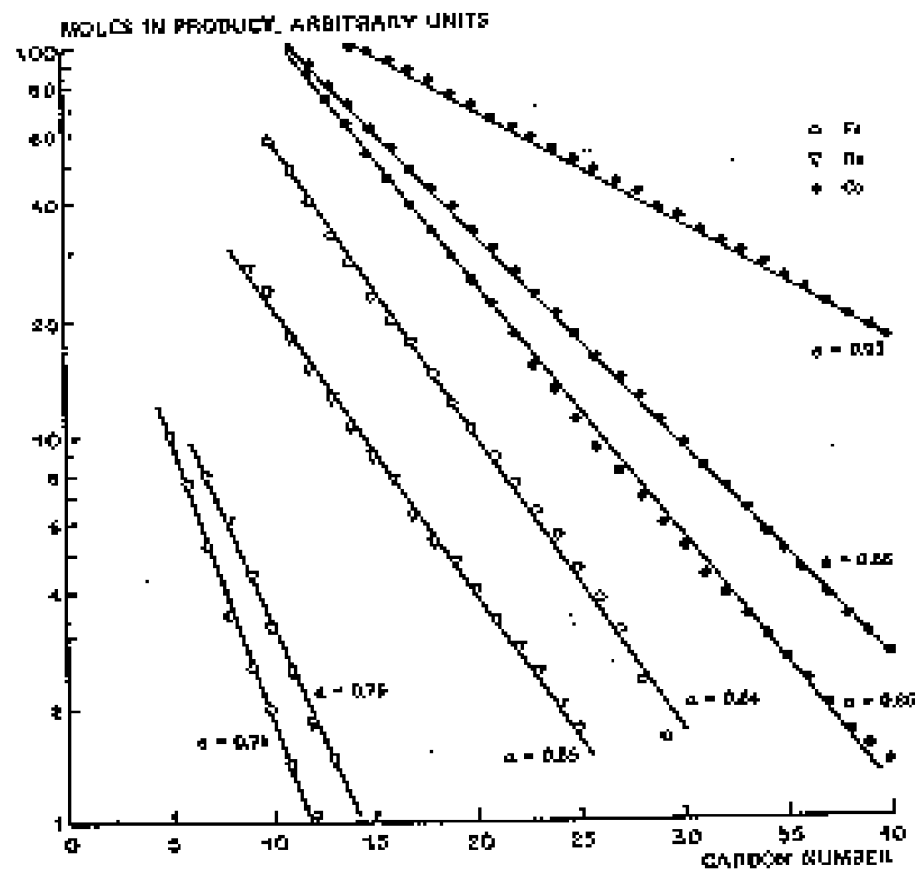

Figure 17. Typseal carbon number dlstribution teported by Stwall tor their FischerTropseh data (from referencos 21\}.

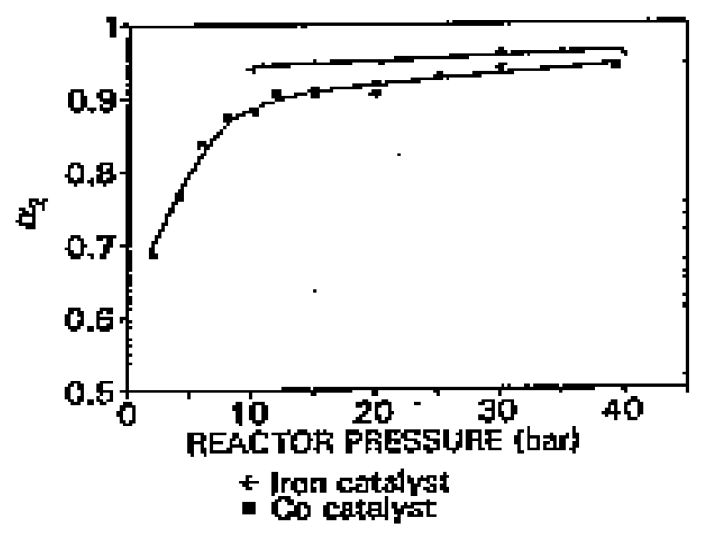

Figure to Chain growth probability $x_{2}$ as a function of reactor pressure at a constant superificial welacity (fiom referenta 14). 

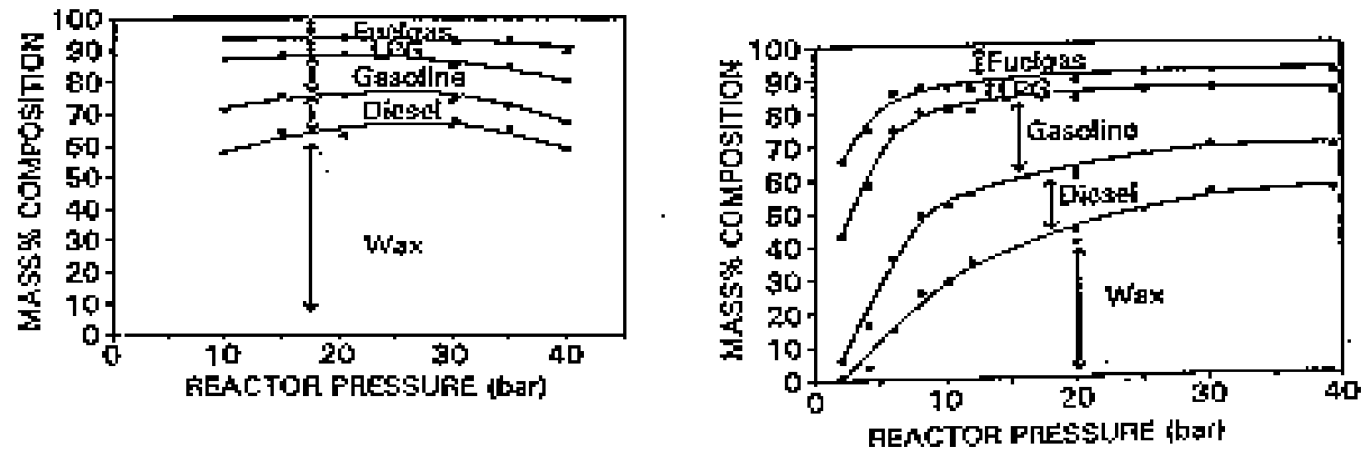

Figure 19. Masox product diatribution ac a function of reacter pressure at oonstart superficial wa|pcily for loft] iron sluriy phase catahyst and [right) a cobelt estabyt ['rom referenta 1.fi].

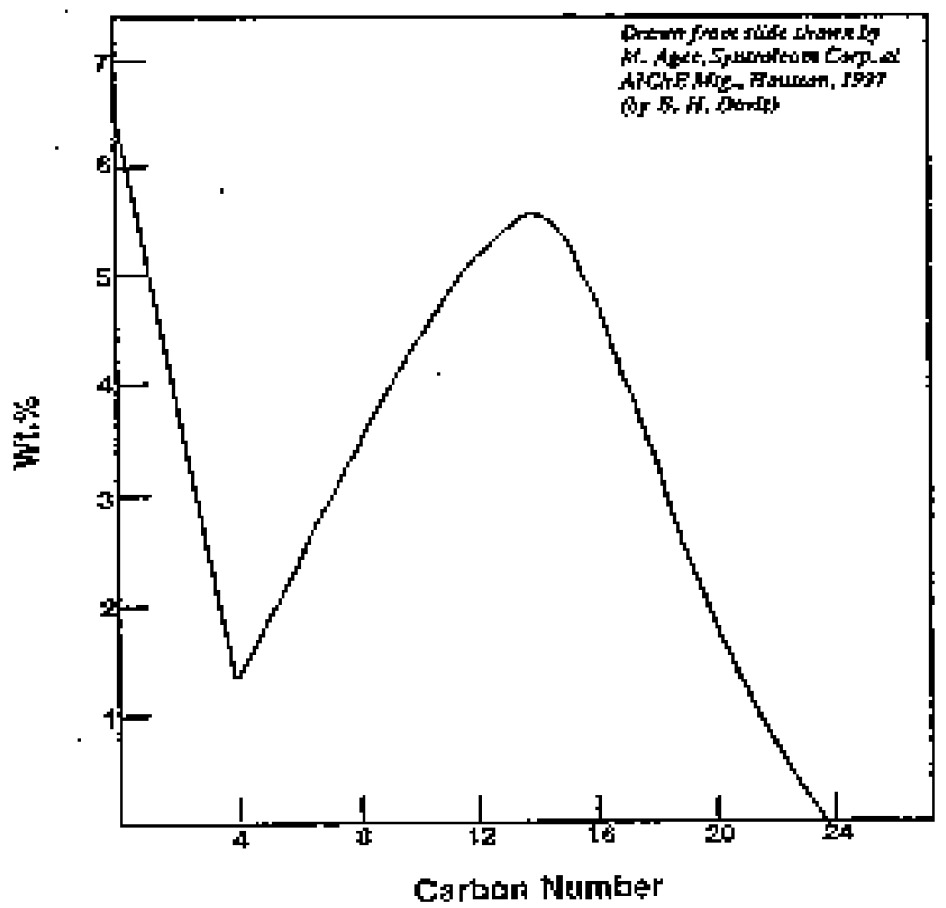

Figure 20. Plot stwring selectivity data for Syntroleum "thair limiling" catahyst [ifrawn from talk given by M, Agee at AlChE Mesting. Holston, 1997). 


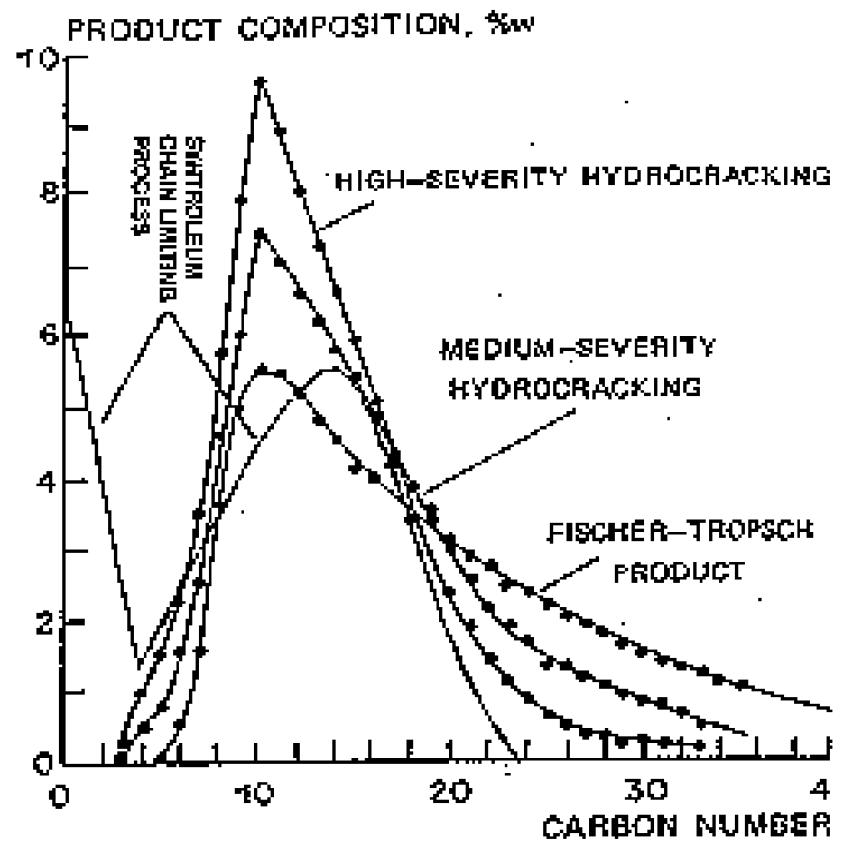

Flgure 21. [ate from fiọur 20 superimposed upon bydropracking data presented by Shell \{from reference 21\},

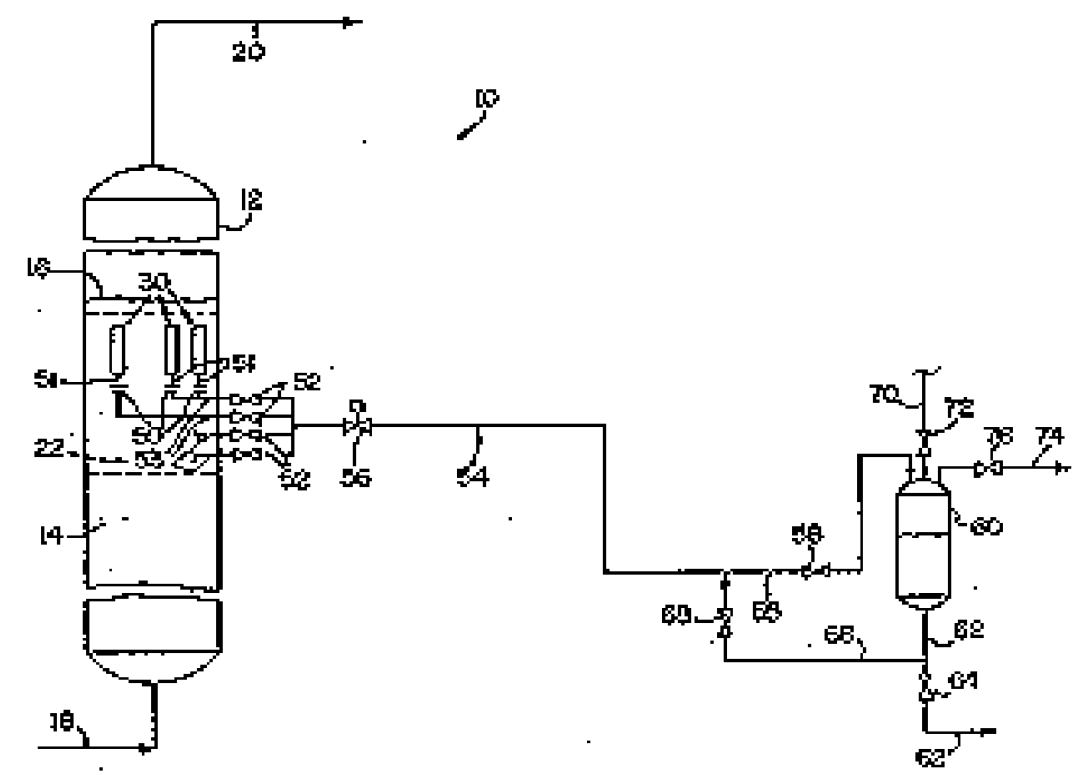

Fgure 2. Schematio of reactor and fltration apparatus patented by Sasol. 

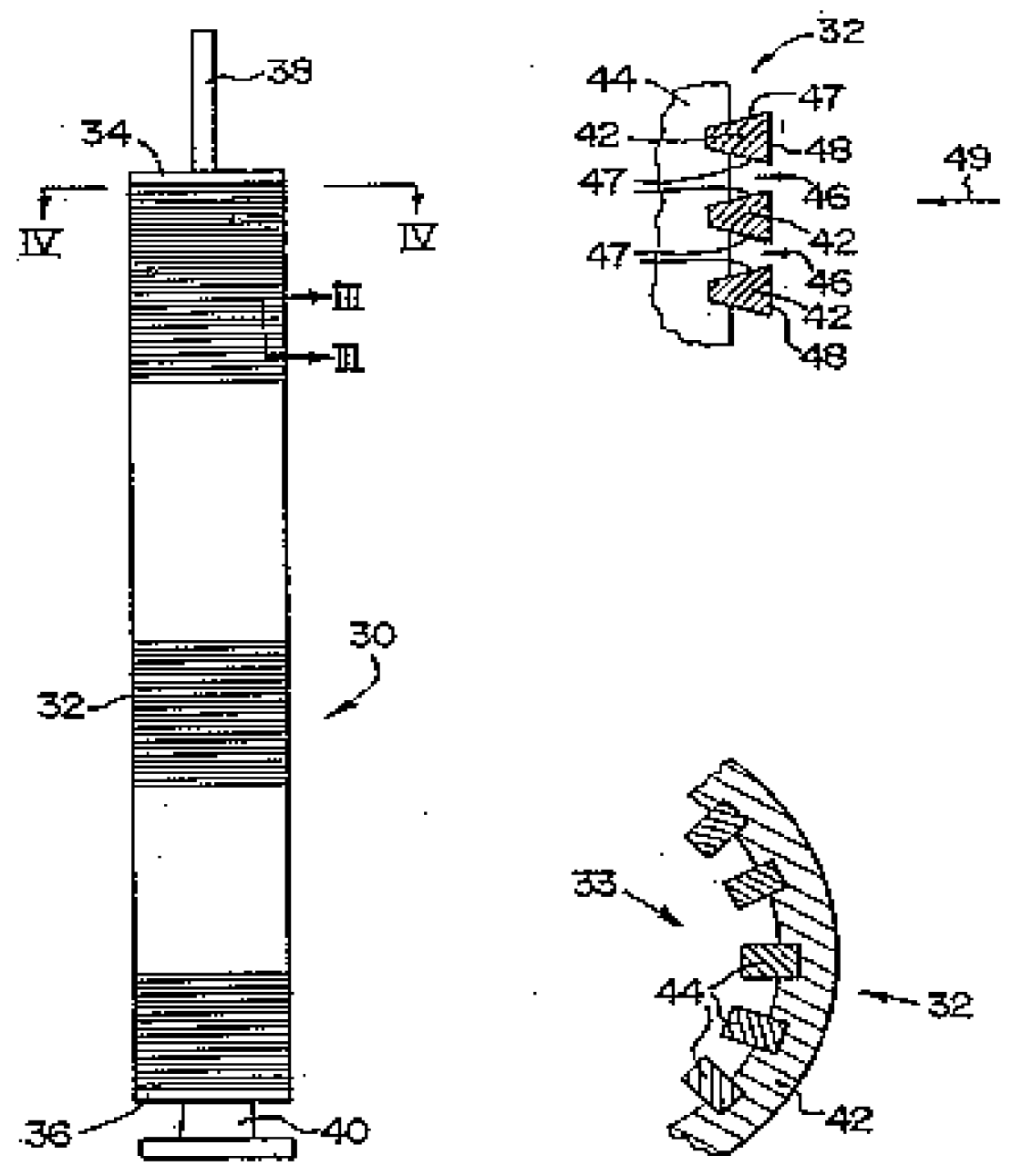

Frgure 29. Expended scale draying of Sasol waycatabst seperation device. 


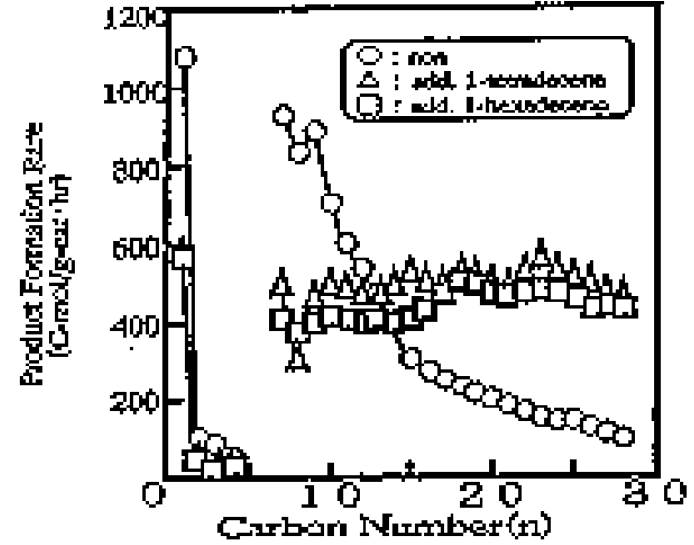

Figure 25. Produgt dlstribution in the olefin-added supercidical-phase F.T, reaction

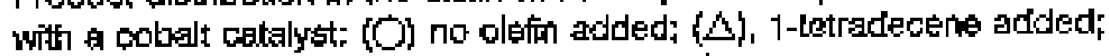
([D] 1-hoxadecene added (from reference 42).
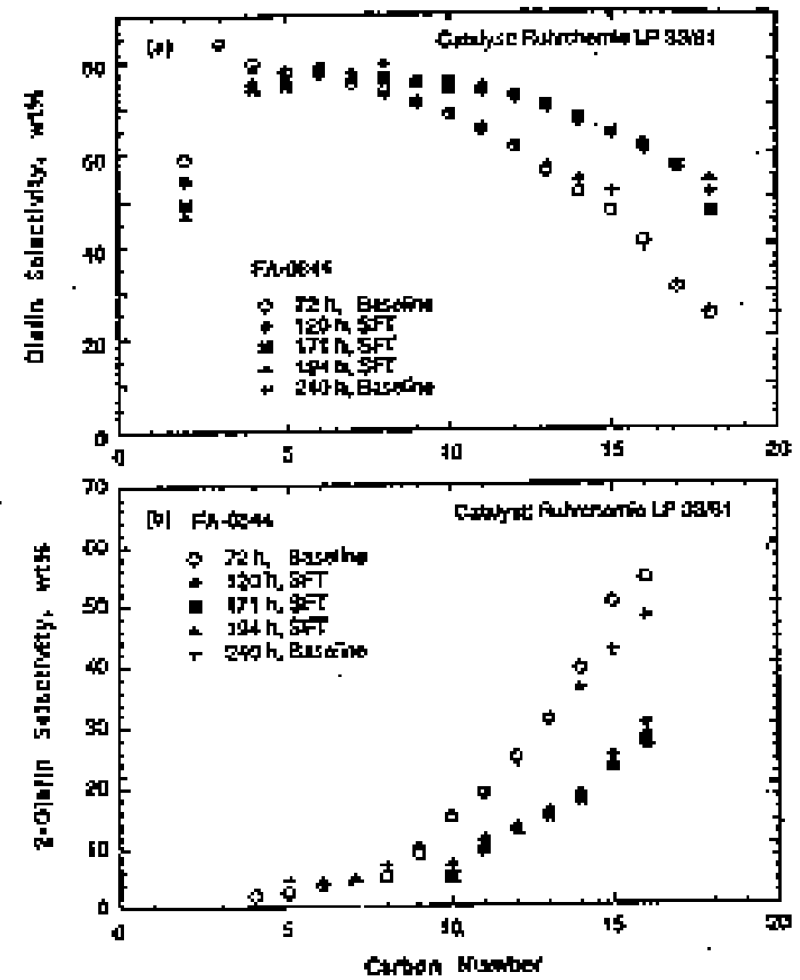

Figlure 26. Effect of supercitical Ft Synthests on a-plefin (a) and a-olefin (b) selectinity using an iron satalyst (from refirince 44\}- 


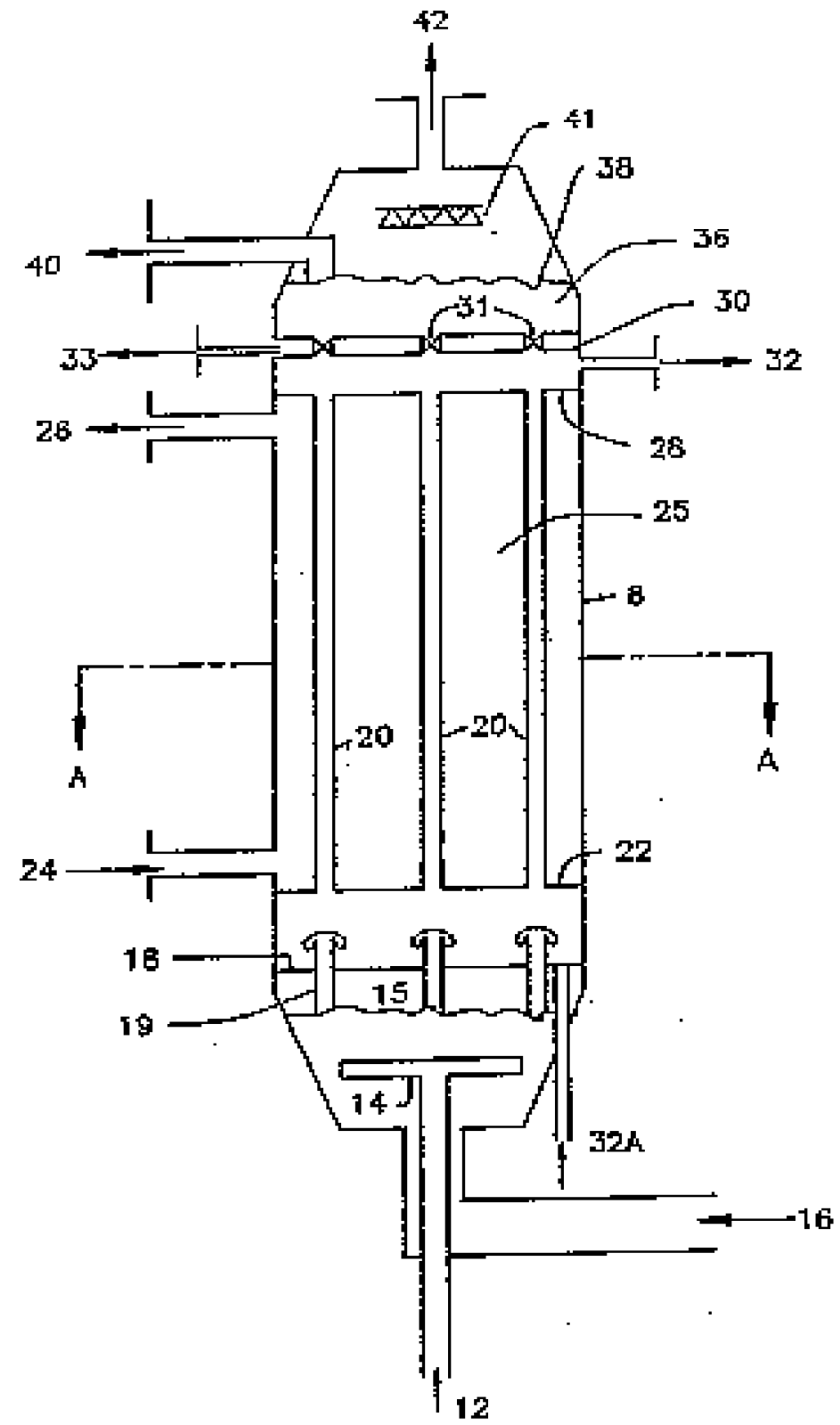

Figlure 24. Schematic of reactor and filiretion apparatus patarlad by Eoon fifom referenco 293. 


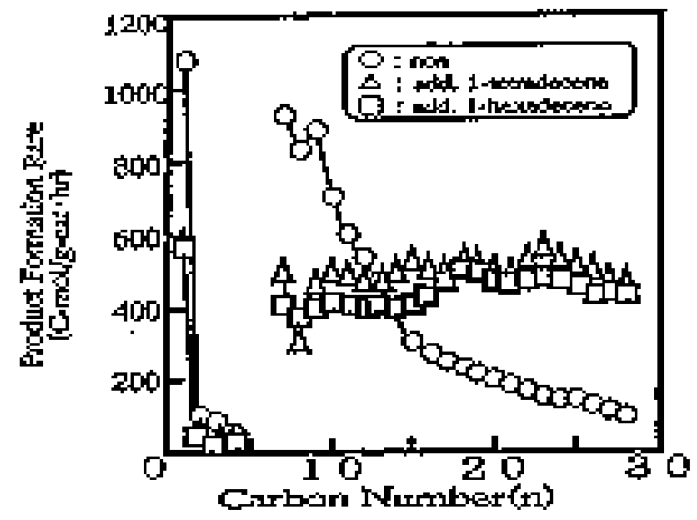

Figure 25. Product dlstribution in the olefin-added superitical-phage F.T. rodetion with a pobalt cotalyst: (C) no oletn added; \{A\}, 1-Letradecene added; ([] 1-hoxadecene added (from reference 4Z) .
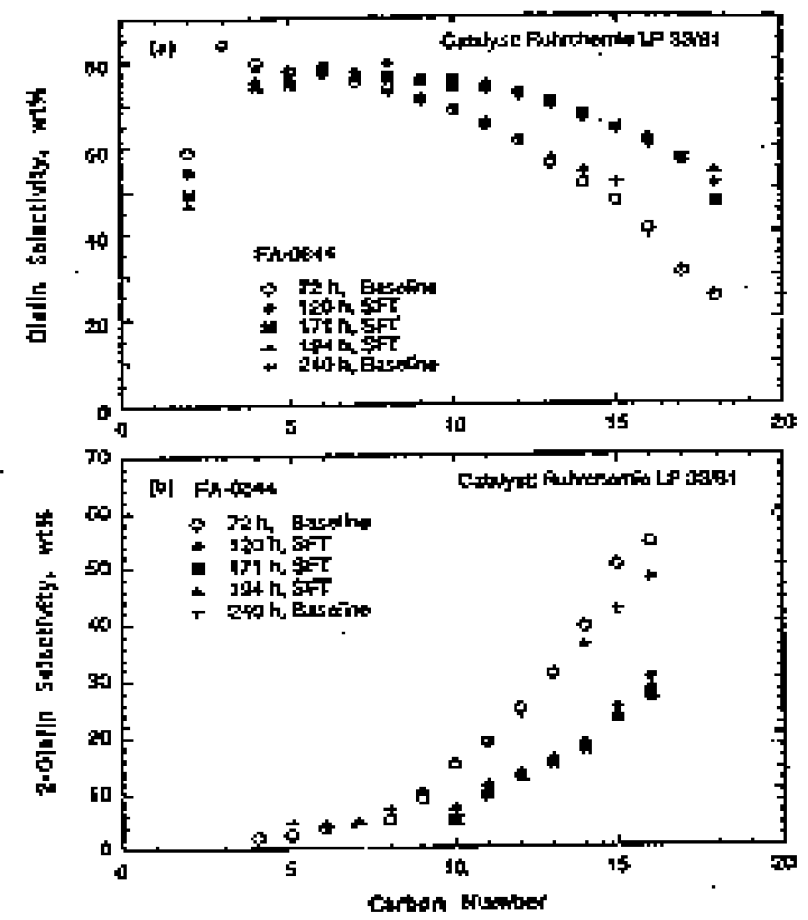

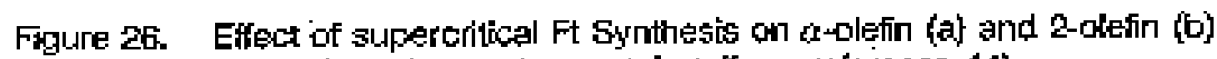
. selectinty using an iron ostalyst (firem referience 44). 